\title{
A Theoretical Performance Analysis and Simulation of Time-Domain EMI Sensor Data for Land Mine Detection
}

\author{
Ping Gao, Student Member, IEEE, and Leslie M. Collins, Member, IEEE
}

\begin{abstract}
In this paper, the physical phenomenology of electromagnetic induction (EMI) sensors is reviewed for application to land mine detection and remediation. The response from time-domain EMI sensors is modeled as an exponential damping as a function of time, characterized by the initial magnitude and decay rate. Currently deployed EMI sensors that are used for the land mine detection process the recorded signal in a variety of ways in order to provide an audio output for the operator to judge whether or not the signal is from a mine. Sensors may sample the decay curve, sum it, or calculate its energy. Based on exponential decay model and the assumption that the sensor response is subject to additive white Gaussian noise, the performance of these, as well as optimal, detectors are derived and compared. Theoretical performance predictions derived using simplifying assumptions are shown to agree closely with simulated performance. It will also be shown that the generalized likelihood ratio test (GLRT) is equivalent to the likelihood ratio test (LRT) for multichannel time-domain EMI sensor data under the additive white Gaussian noise assumption and specific assumptions regarding the statistics of the decay rates of targets and clutter.
\end{abstract}

\section{INTRODUCTION}

I $\mathrm{N}$ order to evaluate the performance of a detection system, there are two fundamental quantities required: the probability of detection $\left(P_{d}\right)$ and the probability of false alarm $\left(P_{f a}\right)$. The receiver operating characteristic (ROC), which is a plot of $P_{d}$ versus $P_{f a}$, is the standard method of illustrating the overall performance of a detector. With the motivation of obtaining quantitative measurements of the performance bounds, and given the fact that experimental minefield data necessarily suffers from a paucity of emplaced targets [1], sensor responses were modeled, and performance was evaluated theoretically and through simulation for a variety of detection algorithms using time-domain electromagnetic induction (EMI) sensor data. The goal of this study was to theoretically compare the performance of algorithms currently implemented in EMI sensors to that of algorithms derived using signal detection theory.

In references [2]-[4], Bayesian decision theoretic approaches to land mine detection using EMI sensor data obtained experimentally in the Defense Advanced Research Projects Agency (DARPA) Backgrounds Clutter Data Collection Experiment [5] were investigated. Two types of time-domain pulsed EMI sys-

Manuscript received March 12, 1999; revised December 2, 1999. This work was supported by Army Research Office Grant DAAH04-96-1-0448 (Demining MURI).

The authors are with the Department of Electrical and Computer Engineering, Duke University, Durham, NC 27708-0291.

Publisher Item Identifier S 0196-2892(00)06221-5. tems were considered: sensors that either sample or integrate the time-domain information to provide a single data point (singlechannel systems) and those that provide a sampled version of the time-domain waveform (multichannel sensors). The standard detection strategy for single-channel time-domain sensor data is simply a threshold test on the raw data recorded from the sensor. Extensions of this approach to multichannel EMI data are a threshold test on the energy present at the location under test, which is called an energy detector, and a threshold test on the integral (sum) of the entire time domain signal, called an integral or summed-data detector. In our previous work, we applied signal detection theory to the DARPA experimental data and incorporated the underlying physics of the sensor [6]-[10] as well as the statistical properties of the responses due to target and clutter/noise to generate the likelihood ratio test (LRT) at each surveyed location. The probability density functions (PDF's) of the responses from mines and clutter/noise were used to formulate the likelihood ratio. However, theoretical performance analyses of these detectors were not performed and the statistical significance of the conclusions which could be drawn based on this preliminary work were limited by the number of targets emplaced in the test sites. In this paper, rigorous theoretical analyses of the performance of the detectors mentioned above will be presented.

When using detection theory to form an LRT, it is sometimes difficult to determine an explicit form for the likelihood ratio since the data is often dependent on one or more unknown parameters. To obtain PDF's of the response, $r$, from target and clutter $\left(p\left(r \mid H_{1}\right)\right.$ and $p\left(r \mid H_{0}\right)$ respectively), one must integrate over these parameters (details shown in Section III), which can be computationally expensive. (Here, $H_{1}$ is the hypothesis that a target (a mine), is present, and $H_{0}$ is the hypothesis that a target is not present, i.e. clutter or noise is present.) To avoid computational complexity, the generalized LRT (GLRT) (see Section III) provides a simplified, yet sub-optimal version of the LRT. In some cases, the GLRT achieves the same performance as the LRT, however, this is not generally the case [11]. In [2], [3], it has been shown that for single-channel EMI data, the optimal processor at each surveyed location is equivalent to a threshold test performed on the raw data under some assumptions on the statistics of the underlying process. In this paper, we show that for multichannel EMI data, the GLRT performed at a single surveyed location is the optimal detector under a similar set of assumptions. We also show that under the necessary assumptions, the form of the GLRT is a filter matched to the difference between the mean responses from targets and clutter. 


\section{SENSOR Phenomenology}

An EMI system is essentially a metal detector. It records the induced electromagnetic field due to an incident electromagnetic field which impinges on underground objects, clutter, etc. An EMI system can detect mines that have metal content, as well as other unexploded ordnance (UXO) or anthropic clutter which contain metal. In order to detect such targets, the EMI system normally operates at low frequencies $(<1 \mathrm{MHz})$, at which the conductivity- and permeability-dependent skin depth of the materials varies significantly [6], [7]. Furthermore, at these frequencies the displacement current is weak enough to be overlooked [12]. Hence, the response of the pulsed EMI system, $\boldsymbol{r}$, at each location surveyed with the sensor can be modeled as a superposition of weighted resonances

$$
r(t)=\sum_{i=1}^{N} A_{n} e^{j \omega_{n} t}
$$

where $\omega_{n}$ is the $n$th natural resonant frequency of the object, and $A_{n}$ is the initial magnitude of the response corresponding to that natural resonant frequency. In practice, the real part of $\omega_{n}$ is very small, and thus can be ignored [12]. The late time field, which is the field recorded by EMI sensors, is usually dominated by the lowest mode. Therefore, only one exponential damping coefficient need be considered. The response can thus be modeled (approximately) as

$$
r(t)=A e^{-\alpha t}
$$

where $A$ is the initial magnitude of the response, and $\alpha$ is the lowest natural resonant frequency. $A$ is strongly dependent on the excitation level, as well as the depth and the orientation of the underground objects. The resonant frequency $\alpha$ is a function of conductivity and permeability. Both theoretical and experimental data demonstrate that $\alpha$ is unique to each metal type [6], [8], [12]. Consequently, it can be used to identify land mines. On average, metal mines have a lower natural resonant frequency than clutter, i.e. the decay rate of the target signature is slower.

In this paper, we consider data from two classes of EMI sensors: sensors that sample or perform some sort of integration on the time-domain information to provide a single data point (standard metal detector) and those that provide a sampled portion of the time-domain waveform. An example of the first system, the Geonics EM61, consists of a single-channel pulsed induction system with a $0.5 \mathrm{~m}$ transmitter coil positioned approximately $0.3 \mathrm{~m}$ above the ground. Data is received in both the transmitter coil and a secondary receiver coil that is located $0.4 \mathrm{~m}$ above the transmitter. The system operates at a center frequency of $75 \mathrm{~Hz}$; the received signal is integrated from 0.18 to $0.87 \mathrm{~ms}$ after each transmitting pulse. The result, a single scalar value, is stored for later processing. Therefore, its response can be expressed as

$$
r=\sum_{i=0}^{N} A e^{-\alpha\left(t_{0}+i \Delta t\right)}
$$

where $t_{0}$ is the initial time for integration and the integration ends at $t_{0}+N \Delta t$. Another example of a single channel system is the standard army device, the PSS-12, which is manufactured by Schiebel. The output of this sensor is a single sample of the time-domain waveform. An example of a multichannel time-domain pulsed EMI sensor is the Geonics EM61-3D sensor. It is a three-component, time-domain induction system having a $1 \mathrm{~m}$ square transmitter coil and three orthogonal $0.5 \mathrm{~m}$ receiver coils positioned approximately $0.3 \mathrm{~m}$ above the ground. The system operates at a center frequency of $7.5 \mathrm{~Hz}$. Sensor output is measured and recorded at 20 geometrically spaced time gates, spanning a time range from $320 \mu$ s to $30 \mathrm{~ms}$ following the incident pulse [5]. Thus, the received signal of Geonics EM61-3D sensor, $\mathbf{r}(\mathbf{t})$, can be expressed as

$$
\mathbf{r}(\mathbf{t})=A e^{-\alpha \mathbf{t}}
$$

where $\mathbf{t}$ is a $20 \times 1$ vector whose elements are the sampling times.

In this work, we have normalized the initial value, $A$, and have thus concentrated on the information conveyed by the resonant frequency $\alpha$. Note that since the actual sensor output is subject to noise, only approximate normalization for real data is possible. Because the SNR at the early time response is high (since the response is essentially an exponential decay), the estimate of $A$ is reasonably accurate.

\section{PERformance AnAlysis}

In our previous work [2]-[4], a set of signal processing algorithms were applied to the EMI data collected in conjunction with the DARPA Background Clutter Data Collection Experiment [5]. However, no theoretical performance analyses and simulations on synthetic data were performed to validate the conclusions from the experimental study. Such analyses are necessary since so few targets were emplaced in the DARPA experiment. In this section, theoretical analyses of the performance for the LRT, the GLRT, the integral/sum, and the energy detectors and single-point detector are investigated. Additionally, when we consider a single time sample of the time-domain EMI response, the sample time that can provide the best performance using this type of data is derived. These results are then verified by simulation (Section IV).

We have assumed that the sensor is subject to independent and identically distributed (IID) Gaussian noise at each time sample. Therefore, the conditional density of $\mathbf{r}\left(r_{1}, r_{2}, \cdots, r_{N}\right)$ is jointly Gaussian. For the Gaussian noise problem, the performance of the detector is completely characterized by the quantity $d^{\prime 2}$ [11], [13], where $d^{\prime}$ is defined as the distance between the means of the two hypotheses when the variance is normalized to one

$$
d^{2} \equiv \frac{\left(E\left(l \mid H_{1}\right)-E\left(l \mid H_{0}\right)\right)^{2}}{\operatorname{Var}\left(l \mid H_{0}\right)}
$$

where $l$ is the output of the processor, which is a function of received signal $\mathbf{r}$, and $E(\cdots)$ and $\operatorname{Var}(\cdots)$ represent the mean and variance. Hence, based on the assumptions stated previously, the $d^{\prime 2}$ value of each detector is derived under a variety of assumptions regarding the amount of information which is known. In Section IV, we compare the ROC for the theoretical calculation of $d^{\prime 2}$ with the simulation results. 


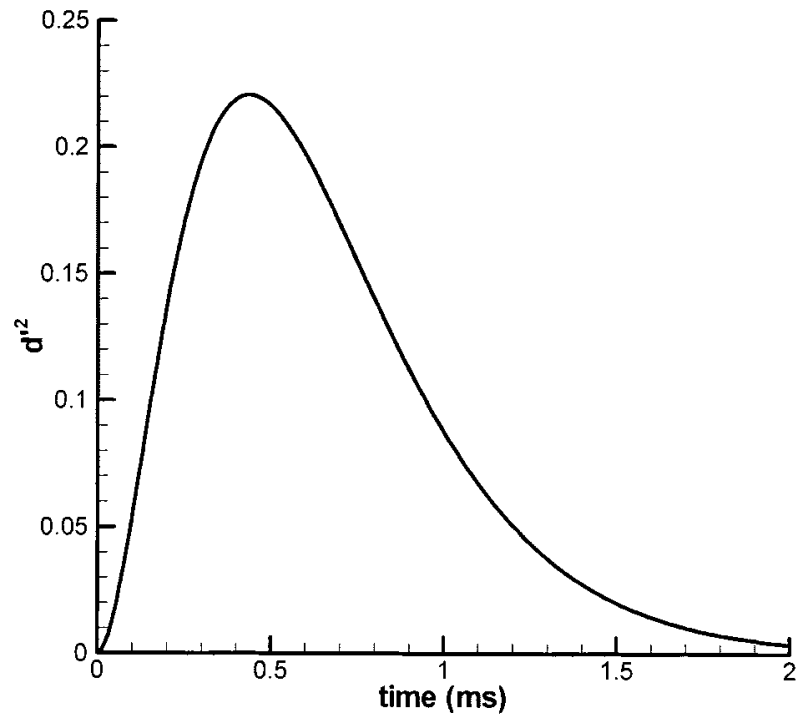

Fig. 1. $d^{2}$ as a function of sample time $t_{s}$ when parameters are fixed and known values. $\alpha_{1}=2.1, \alpha_{0}=2.5$, and $\sigma=0.14$.

\section{A. Fixed Parameter Case}

As described previously, the output from the EMI sensor depends on whether or not a mine is present at the location under test. It is a function of the noise variance $\sigma^{2}$ and the decay rate $\alpha_{1}$ for $H_{1}$, and $\alpha_{0}$ for $H_{0}$. There are also other factors, such as temperature, humidity, environmental noise, etc., which affect the response. In this study, we have not considered these additional parameters. If the parameters $\alpha_{1}, \alpha_{0}$, and $\sigma^{2}$ are known values or can be estimated (by MLE, MMSE, etc.) the $d^{\prime 2}$ corresponding to single time sample data, the LRT, an integral, and an energy detector on multichannel time-domain EMI data can be calculated as follows.

1) Single Time Sample of Time Domain (TD) EMI Data: If a time-domain EMI sensor samples the induced response at a single point in time, the performance of this sensor depends on the sample time used by the sensor. Thus, in order to achieve the best performance under the physical constraints imposed by the sensor, the time at which a sample is taken after the incident pulse vanishes becomes a key issue.

It is assumed that the sensor is subject to additive white Gaussian noise, i.e. $\mathcal{N}\left(0, \sigma^{2}\right)$. From detection theory, we know that the optimal detector operating on this type of data is simply a threshold test. Therefore

$$
d^{\prime 2}=\frac{\left(e^{-\alpha_{1} t_{s}}-e^{-\alpha_{0} t_{s}}\right)^{2}}{\sigma^{2}}
$$

where $t_{s}$ is the operating sample time of the sensor. Fig. 1 plots $d^{\prime 2}$ as a function of sample time $t_{s}$. To determine the sample time that maximizes $d^{\prime}$, the derivative of $d^{\prime}$ with respect to $t_{s}$ is taken and set to zero. The sample time maximizing $d^{\prime}$ is

$$
t_{\max }=\frac{\ln \alpha_{0}-\ln \alpha_{1}}{\alpha_{0}-\alpha_{1}}
$$

where $\ln (-)$ is the natural logarithm. Note that the best sample time is a function of the decay rates of the target and clutter, so it is object dependent.
2) LRT (Matched Filter) on Multichannel TD EMI Data: The LRT is defined as $\lambda(r) \equiv\left(p\left(r \mid H_{1}\right) / p\left(r \mid H_{0}\right)\right)$ [11], [13], [14], where $r$ is the sensor output, $H_{1}$ is the target present hypothesis, and $H_{0}$ is the no-target hypothesis. If the decay rates for targets and clutter $\alpha_{1}$ and $\alpha_{0}$ are known constants, from the definition of the LR, the LRT reduces to a matched filter $y=\sum_{i} r_{i}\left(e^{-\alpha_{1} t_{i}}-e^{-\alpha_{0} t_{i}}\right)$, where in this case, the data is matched to the difference of the two main signals. This is a signal known exactly (SKE) case, and the LR is distributed as a Gaussian random variable, i.e. under $H_{1}, y \sim \mathcal{N}\left(\mu_{1}, \sigma_{1}^{2}\right)$, and under $H_{0}, y \sim \mathcal{N}\left(\mu_{0}, \sigma_{0}^{2}\right)$, where

$$
\begin{aligned}
\mu_{1} & =\sum_{i}\left(e^{-\alpha_{1} t_{i}}-e^{-\alpha_{0} t_{i}}\right) e^{-\alpha_{1} t_{i}}, \\
\sigma_{i}^{2} & =\sum_{i}\left(e^{-\alpha_{1} t_{i}}-e^{-\alpha_{0} t_{i}}\right)^{2} \sigma^{2}, \\
\mu_{0} & =\sum_{i}\left(e^{-\alpha_{1} t_{i}}-e^{-\alpha_{0} t_{i}}\right) e^{-\alpha_{0} t_{i}}
\end{aligned}
$$

and $\sigma_{0}^{2}=\sigma_{1}^{2}$. Thus

$$
d^{\prime 2}=\frac{\left(\mu_{1}-\mu_{0}\right)^{2}}{\sigma_{0}^{2}}=\frac{\sum_{i}\left(e^{-\alpha_{1} t_{i}}-e^{-\alpha_{0} t_{i}}\right)^{2}}{\sigma^{2}}
$$

As expected, $d^{\prime}$ is proportional to the energy of the difference signal and inversely proportional to the noise variance.

3) Integral/Summed-Data Detector on Multichannel TD EMI Data: An integral detector, also called a summed-data detector, integrates (sums) the sampled time sequence, then compares the sum to a threshold to make a decision as to whether a mine is present or not. The output of an integral detector is $x=\Sigma r_{i}$ and thus under $H_{1}, x \sim \mathcal{N}\left(\sum e^{-\alpha_{1} t_{i}}\right.$, $\left.N \sigma^{2}\right)$ and under $H_{0}, x \sim \mathcal{N}\left(\sum e^{-\alpha_{0} t_{i}}, N \sigma^{2}\right)$. Hence

$$
d^{\prime 2}=\frac{\left[\sum_{i}\left(e^{-\alpha_{1} t_{i}}-e^{-\alpha_{0} t_{i}}\right)\right]^{2}}{N \sigma^{2}}
$$

4) Energy Detector on Multichannel TD EMI Data: The energy detector compares the energy $\left(E=\Sigma r_{i}^{2}\right)$ to a threshold to make a decision. Based on the assumptions made before, under $H_{1}$

$$
\begin{aligned}
E & =\sum_{i}\left(e^{-\alpha_{1} t_{i}}+n_{i}\right)^{2} \\
& =\sum_{i} e^{-2 \alpha_{1} t_{i}}+2 \sum_{i} e^{-\alpha_{1} t_{i}} n_{i}+\sum_{i} n_{i}^{2} .
\end{aligned}
$$

The first term of the right-hand side of (10) is a constant, the second term is a Gaussian distributed random variable with mean of zero and variance of $4 \sigma^{2} \sum e^{-\alpha_{i} t_{i}}$, and the third term is distributed as $\sigma^{2} \chi_{N}^{2}$ [16], where $\chi_{N}^{2}$ is a chi-square distribution with $N$ degrees of freedom. Based on the central limit theorem, the third term can be approximated by a Gaussian random 
variable with a mean of $N \sigma^{2}$ and a variance of $2 N \sigma^{4}$. The correlation between the second and the third terms in (10) is

$$
\begin{aligned}
& E\left[\sum_{i}\left(e^{-\alpha_{1} t_{i}} n_{i}\right)\left(\sum n_{i}^{2}\right)\right] \\
& \quad=E\left[\left(\sum_{i} e^{-\alpha_{1} t_{i}} n_{i}\right)\left(n_{1}^{2}+n_{2}^{2}+\cdots+n_{N}^{2}\right)\right] \\
& \quad=\sum_{i} E\left[\left(e^{-\alpha_{1} t_{i}} n_{i}\right)\left(n_{1}^{2}+n_{2}^{2}+\cdots+n_{N}^{2}\right)\right] .
\end{aligned}
$$

Since $n_{i}$ and $n_{j}$ are independent, if $i \neq j, E\left[n_{i} n_{j}^{2}\right]=$ $E\left[n_{i}\right] E\left[n_{j}^{2}\right]=0$, and $E\left[n_{i}^{3}\right]=0$ (since the mean of the odd power of a zero-mean Gaussian random variable is zero), then

$$
\begin{gathered}
E\left[\sum_{i} e^{-\alpha_{1} t_{i}} n_{i}\right] E\left[\left(\sum_{i} n_{i}^{2}\right)\right] \\
=\left(\sum_{i} e^{-\alpha_{1} t_{i}} E\left[n_{i}\right]\right) E\left[\sum_{i} n_{i}^{2}\right]=0 . \\
E\left[\left(\sum_{i} e^{-\alpha_{1} t_{i}} n_{i}\right)\left(\sum n_{i}^{2}\right)\right]=0 .
\end{gathered}
$$

Therefore, the correlation between the second and the third terms of (10) is zero, implying that they are independent. since they are (approximately) Gaussian distributed [15]. Thus, the energy under $H_{1}$ can be approximated by a Gaussian distribution with a mean of $\sum e^{-2 \alpha_{1} t_{i}}+N \sigma^{2}$ and variance $4 \sigma^{2} \sum e^{-2 \alpha_{1} t_{i}}+2 N \sigma^{4}$. Similarly, under $H_{0}$. the energy also follows a Gaussian distribution with a mean of $\sum e^{-2 \alpha_{0} t_{i}}+N \sigma^{2}$ and variance $4 \sigma^{2} \sum e^{-2 \alpha_{0} t_{i}}+2 N \sigma^{4}$. Therefore

$$
\begin{aligned}
d^{\prime 2} & =\frac{\left(\mu_{1}-\mu_{0}\right)^{2}}{\left(\sigma_{1}^{2}+\sigma_{0}^{2}\right) / 2} \\
& =\frac{\left[\sum_{i}\left(e^{-2 \alpha_{1} t_{i}}-e^{-2 \alpha_{0} t_{i}}\right)\right]^{2}}{2 \sigma^{2}\left[\sum_{i} e^{-2 \alpha_{1} t_{i}}+e^{-2 \alpha_{0} t_{i}}\right]+2 N \sigma^{4}} .
\end{aligned}
$$

\section{B. Random Parameter Case}

In practice, the decay rates of both targets and clutter are not known constants, but vary within some range. Without compromising generality and for simplicity of further calculation, a reasonable assumption on the distribution of $\alpha_{1}$ and $\alpha_{0}$ is Gaussian. Furthermore, $\alpha_{1}$ and $\alpha_{0}$ are independent of additive sensor noise, since they are intrinsic properties of the objects being considered. Practically, the decay rate cannot be negative, thus, the distributions of $\alpha_{1}$ and $\alpha_{0}$ can be considered as truncated Gaussian distributions

$$
\begin{aligned}
p\left(\alpha_{1}\right)= & \left(\Phi\left(\frac{\alpha_{1}^{u}-\mu_{\alpha_{1}}}{\sigma_{\alpha_{1}}}\right)-\Phi\left(\frac{\alpha_{1}^{l}-\mu_{\alpha_{1}}}{\sigma_{\alpha_{1}}}\right)\right)^{-1} \\
& \cdot \frac{1}{\sqrt{2 \pi \sigma_{\alpha_{1}}^{2}}} e^{-\left(\alpha_{1}-\mu_{\alpha_{1}}\right)^{2} / 2 \sigma_{\alpha_{1}}^{2}}
\end{aligned}
$$

and

$$
\begin{aligned}
p\left(\alpha_{0}\right)= & \left(\Phi\left(\frac{\alpha_{0}^{u}-\mu_{\alpha_{0}}}{\sigma_{\alpha_{0}}}\right)-\Phi\left(\frac{\alpha_{0}^{l}-\mu_{\alpha_{0}}}{\sigma_{\alpha_{0}}}\right)\right)^{-1} \\
& \cdot \frac{1}{\sqrt{2 \pi \sigma_{\alpha_{0}}^{2}}} e^{-\left(\alpha_{0}-\mu_{\alpha_{0}}\right)^{2} / 2 \sigma_{\alpha_{0}}^{2}}
\end{aligned}
$$

where

$$
\Phi(x)=\frac{1}{\sqrt{2 \pi}} \int_{-\infty}^{x} e^{-t^{2} / 2} d t
$$

$\mu_{\alpha_{1}}, \alpha_{\alpha_{1}}^{2}$ and $\mu_{\alpha_{0}}, \sigma_{\alpha_{0}}^{2}$ are the mean and the variance of $\alpha_{1}$ and $\alpha_{0}$, respectively, and $\alpha_{1}^{u}, \alpha_{1}^{l}$, and $\alpha_{0}^{u}, \alpha_{0}^{l}$ are the left and right truncated points of $\alpha_{1}$ and $\alpha_{0}$, respectively. $\alpha_{1}^{l}=\alpha_{0}^{l}=0$ and $\alpha_{1}^{u} \leq \alpha_{0}^{u}$ based on the underlying physics illustrated in Section II. The limits of $\alpha_{1}^{u}, \alpha_{0}^{u}$ are infinity. Based on the field data collected in the DARPA experiment [5], $\Phi\left(\alpha_{1}^{u}-\mu_{\alpha_{1}} / \sigma_{\alpha_{1}}\right)-$ $\Phi\left(\alpha_{1}^{i}-\mu_{\alpha_{1}} / \sigma_{\alpha_{1}}\right)$, and $\Phi\left(\alpha_{0}^{u}-\mu_{\alpha_{0}} / \sigma_{\alpha_{0}}\right)-\Phi\left(\alpha_{0}^{l}-\mu_{\alpha_{0}} / \sigma_{\alpha_{0}}\right)$ are very close to 1 .

The LR test with uncertain parameters is defined as [11], [13], [14]

$$
\lambda(r)=\frac{\int p\left(r \mid H_{1}, \theta_{1}\right) p\left(\theta_{1} \mid H_{1}\right) d \theta_{1}}{\int p\left(r \mid H_{0}, \theta_{0}\right) p\left(\theta_{0} \mid H_{0}\right) d \theta_{0}} \underset{H_{0}}{\stackrel{H_{1}}{\gtrless}} \gamma
$$

where $r$ is the measured, or available, data (which can be a scalar or a vector), $H_{1}$ and $H_{0}$ are the hypotheses of target present and target not present, respectively, $p\left(r \mid H_{1}, \theta_{1}\right)$ and $p\left(r \mid H_{0}, \theta_{0}\right)$ are the PDF's describing the statistical nature of the response, $r$, given hypotheses $\left(H_{1}\right.$ or $\left.H_{0}\right)$, and parameters $\left(\theta_{1}\right.$ or $\left.\theta_{0}\right)$. $\theta_{1}$ and $\theta_{0}$ are unknown parameter sets associated with each hypothesis which follow the distributions $p\left(\theta_{1} \mid H_{1}\right)$ and $p\left(\theta_{0} \mid H_{0}\right)$. In this problem, $\theta_{1}$ and $\theta_{0}$ correspond to the decay rate under the two hypotheses, respectively. When the LR is greater than a threshold, $\gamma, H_{1}$ is chosen, i.e. the target is present. Otherwise, $H_{0}$ is chosen, i.e. no target is present at the test location.

For a more general distribution of $\alpha_{1}$ and $\alpha_{0}$, no quantity such as $d^{\prime 2}$ can easily be derived to characterize the performance. However, an ROC can be obtained through simulation using synthetic data. The noise variance $\sigma^{2}$ is not considered to be a random parameter.

1) GLRT/Matched Filter: The GLRT simplifies the calculation of a test statistic. Instead of integrating over the $\theta_{1}$ and $\theta_{0}$ parameters, as shown in (15), it is defined as [11]

$$
\Omega(r) \equiv \frac{p\left(r \mid H_{1}, \hat{\theta}_{1}\right)}{p\left(r \mid H_{0}, \hat{\theta}_{0}\right)} \underset{H_{0}}{\gtrless} \gamma
$$


where $r$ is defined as before, and $\hat{\theta}_{1}$ and $\hat{\theta}_{0}$ are the maximum likelihood estimates (MLE) of the parameters $\theta_{1}$ and $\theta_{0}$. Based on the assumptions made before, the generalized LR is

$$
\begin{aligned}
\Omega= & \frac{\left(2 \pi \sigma^{2}\right)^{-N / 2} \exp \left[\frac{1}{2 \sigma^{2}} \sum_{i}\left(r_{i}-e^{-\hat{\mu}_{\alpha_{1}} t_{i}}\right)^{2}\right]}{\left(2 \pi \sigma^{2}\right)^{-N / 2} \exp \left[\frac{1}{2 \sigma^{2}} \sum_{i}\left(r_{i}-e^{-\hat{\mu}_{\alpha_{0}} t_{i}}\right)^{2}\right]} \\
= & \exp \left[\frac { 1 } { 2 \sigma ^ { 2 } } \sum _ { i } \left[2 r_{i}\left(e^{-\hat{\mu}_{\alpha_{0}} t_{i}}-e^{-\hat{\mu}_{\alpha_{1}} t_{i}}\right)\right.\right. \\
& \left.+e^{-2 \hat{\mu}_{\alpha_{1}} t_{i}}+e^{-2 \hat{\mu}_{\alpha_{0}} t_{i}}\right]
\end{aligned}
$$

where $\hat{\mu}_{\alpha_{1}}$ and $\hat{\mu}_{\alpha_{0}}$ are estimates of $\mu_{\alpha_{1}}$ and $\mu_{\alpha_{0}}$. ML estimates were used in this study. By taking the logarithm and incorporating the constant into the threshold, the log-GLR is

$$
y=\sum_{i} r_{i}\left(e^{-\hat{\mu}_{\alpha_{1}} t_{i}}-e^{-\hat{\mu}_{\alpha_{0}} t_{i}}\right) .
$$

This is essentially a matched filter, which is matched to the difference between the target response and the clutter response at the estimated decay rates.

Thus

$$
\begin{aligned}
y \mid H_{1}= & \sum_{i}\left(e^{-\alpha_{1} t_{i}}+n_{i}\right)\left(e^{-\hat{\mu}_{\alpha_{1}} t_{i}}-e^{-\hat{\mu}_{\alpha_{0}} t_{i}}\right) \\
= & \sum_{i} e^{-\alpha_{1} t_{i}}\left(e^{-\hat{\mu}_{\alpha_{1}} t_{i}}-e^{-\hat{\mu}_{\alpha_{0}} t_{i}}\right) \\
& +\sum_{i} n_{i}\left(e^{-\hat{\mu}_{\alpha_{1}} t_{i}}-e^{-\hat{\mu}_{\alpha_{0}} t_{i}}\right) .
\end{aligned}
$$

When the variance of $\alpha_{1}$ is relatively small compared to the variance of the noise (this often is the case, as has been verified experimentally [3]), $e^{-\alpha_{1} t_{i}}$ can be approximated by a straight line through the mean value of $\alpha_{1}$ with a slope of the derivative of $e^{-\alpha_{1} t_{i}}$ at $\hat{\mu}_{\alpha_{1}}$, i.e.

$$
e^{-\alpha_{1} t_{i}} \approx-t_{i} e^{-\hat{\mu}_{\alpha_{1}} t_{i}} \alpha_{1}+e^{-\hat{\mu}_{\alpha_{1}} t_{i}}+t_{1} e^{-\hat{\mu}_{\alpha_{1}} t_{i}} \hat{\mu}_{\alpha_{1}} .
$$

Thus, by substituting (20) into (19), $y$ is seen to be a linear combination of Gaussian-distributed random variables. Therefore, $y \sim \mathcal{N}\left(\mu_{1 y}, \operatorname{var}\left(y_{1}\right)\right)$, where

$$
\begin{aligned}
\mu_{1 y}= & \sum_{i} e^{-\hat{\mu}_{\alpha_{1}} t_{i}}\left(e^{-\hat{\mu}_{\alpha_{1}} t_{i}}-e^{-\hat{\mu}_{\alpha_{0}} t_{i}}\right) \\
\operatorname{var}\left(y_{1}\right)= & \sum_{i}\left[\left(e^{-\hat{\mu}_{\alpha_{1}} t_{i}}-e^{-\hat{\mu}_{\alpha_{0}} t_{i}}\right) t_{i} e^{-\hat{\mu}_{\alpha_{1}} t_{i}}\right]^{2} \sigma_{\alpha_{1}}^{2} \\
& +\sum_{i}\left(e^{-\hat{\mu}_{\alpha_{1}} t_{i}}-e^{-\hat{\mu}_{\alpha_{0}} t_{i}}\right)^{2} \sigma^{2} .
\end{aligned}
$$

Similarly, $y \mid H_{0} \sim \mathcal{N}\left(\mu_{0 y}, \operatorname{var}\left(y_{0}\right)\right)$, where

$$
\begin{aligned}
\mu_{0 y}= & \sum_{i} e^{-\hat{\mu}_{\alpha_{0}} t_{i}}\left(e^{-\hat{\mu}_{\alpha_{1}} t_{i}}-e^{-\hat{\mu}_{\alpha_{0}} t_{i}}\right) \\
\operatorname{var}\left(y_{0}\right)= & \sum_{i}\left[\left(e^{-\hat{\mu}_{\alpha_{1}} t_{i}}-e^{-\hat{\mu}_{\alpha_{0}} t_{i}}\right) t_{i} e^{-\hat{\mu}_{\alpha_{0}} t_{i}}\right]^{2} \sigma_{\alpha_{0}}^{2} \\
& +\sum_{i}\left(e^{-\hat{\mu}_{\alpha_{1}} t_{i}}-e^{-\hat{\mu}_{\alpha_{0}} t_{i}}\right)^{2} \sigma^{2} .
\end{aligned}
$$

Therefore, $d^{\prime 2}$ is as shown in (23), at the bottom of the page.

It is shown in Section IV that the LRT reduces to the GLRT for EMI data. Therefore, no additional analysis is required to determine the performance of the LRT.

2) Integral/Summed-Data Detector: Letting $x=\Sigma r_{i}$ correspond to an integral detector output, and using the approximation expressed in (20), then under $H_{1}, x=\sum_{i} e^{-\alpha_{1} t_{i}}+n_{i}$, so

$$
x \mid H_{1} \sim N\left(\sum e^{-\hat{\mu}_{\alpha_{1}} t_{i}},\left(\sum t_{i} e^{-\hat{\mu}_{\alpha_{1}} t_{i}}\right)^{2} \sigma_{\alpha_{1}}^{2}+N \sigma^{2}\right) .
$$

Similarly,

$$
x \mid H_{0} \sim N\left(\sum e^{-\hat{\mu}_{\alpha_{0}} t_{i}},\left(\sum t_{i} e^{-\hat{\mu}_{\alpha_{0}} t_{i}}\right)^{2} \sigma_{\alpha_{0}}^{2}+N \sigma^{2}\right) .
$$

Hence, the result for $d^{2}$ is (26), as shown at the bottom of the next page.

3) Energy Detector: The output of an energy detector on EMI data is either $x=\sum_{i}\left(e^{-\alpha_{1} t_{i}}+n_{i}\right)^{2}$ under $H_{1}$, or $\sum_{i}\left(e^{-\alpha_{0} t_{i}}+n_{i}\right)^{2}$ under $H_{0}$. Since both $\alpha_{1}$ and $\alpha_{0}$ are random variables, the distribution of the energy cannot be accurately approximated by a Gaussian random variable. Therefore, it is not valid to calculate $d^{\prime}$, since the definition of $d^{\prime}$ is based on Gaussian-distributed data. Even though $d^{\prime}$ is not applicable, we can explain the fact that an energy detector exhibits better performance than an integral detector. Since the noise is IID at each sample time, the noise variance at each sample of the sensor is

$$
\begin{aligned}
d^{\prime 2} & =\frac{\left(\mu_{1}-\mu_{0}\right)^{2}}{\left(\sigma_{1}^{2}+\sigma_{0}^{2}\right) / 2} \\
& =\frac{\left[\sum\left(e^{-\hat{\mu}_{\alpha_{1}} t_{i}}-e^{-\hat{\mu}_{\alpha_{0}} t_{i}}\right)\right]^{2}}{\frac{1}{2}\left(\sum_{i} t_{i}^{2}\left(e^{-\hat{\mu}_{\alpha_{1}} t_{i}}-e^{-\hat{\mu}_{\alpha_{0}} t_{i}}\right)^{2}\left(e^{-2 \hat{\mu}_{\alpha_{1}} t_{i}} \sigma_{\alpha_{1}}^{2}-e^{-2 \hat{\mu}_{\alpha_{0}} t_{i}} \sigma_{\alpha_{0}}^{2}\right)\right)+\sum_{i}\left(e^{-\hat{\mu}_{\alpha_{1}} t_{i}}-e^{-\hat{\mu}_{\alpha_{0}} t_{i}}\right)^{2} \sigma^{2}} .
\end{aligned}
$$


the same. For lower-level signals, corresponding to later samples, the SNR is lower than that for higher-level signals. Because the operation of calculating the energy puts more weight on higher values and less weight on lower signals. When the value of the signal is greater than 1, lower SNR time samples contribute less, and consequently, the noise affects the results of the energy detector less than the integral detector, which assigns equal weights to each time sample.

4) Single Time Sample of TD EMI Data: If the decay rate of target or clutter is not a known constant, $d^{\prime}$ is more complicated. Borrowing the result of Section IV, the LRT and GLRT is equivalent for TD EMI data under some specific assumptions. Hence, using the approximation in (20), it can be shown that

$$
d^{\prime 2}=\frac{\left(e^{-\hat{\mu}_{\alpha_{1}} t_{s}}-e^{-\hat{\mu}_{\alpha_{0}} t_{s}}\right)^{2}}{\sigma^{2}+\frac{t^{2}}{2}\left[e^{-2 \hat{\mu}_{\alpha_{1}} t_{s}} \sigma_{\alpha_{1}}^{2}+e^{-2 \hat{\mu}_{\alpha_{0}} t_{s}} \sigma_{\alpha_{0}}^{2}\right]} .
$$

It is difficult to obtain an analytical expression of the sample time that maximizes $d^{\prime}$, since it involves transcendental function. However, it is easy to find the maximum numerically. If $\sigma_{\alpha_{1}}^{2} \ll 1, \sigma_{\alpha_{0}}^{2} \ll 1$, then

$$
d^{\prime 2} \approx \frac{\left(e^{-\hat{\mu}_{\alpha_{1}} t_{s}}-e^{-\hat{\mu}_{\alpha_{0}} t_{s}}\right)^{2}}{\sigma^{2}}
$$

So

$$
t_{\max } \approx \frac{\ln \hat{\mu}_{\alpha_{0}}-\ln \hat{\mu}_{\alpha_{1}}}{\hat{\mu}_{\alpha_{0}}-\hat{\mu}_{\alpha_{1}}}
$$

\section{EQUIVALENCE OF THE GLRT AND THE LRT FOR Multi-CHANNEL EMI DATA}

In many applications, the GLRT is often used instead of the LRT to reduce computational complexity at the cost of sacrificing performance. However, it is not in general an optimal processor [11]. In the following analyses, we show that under some reasonable assumptions made for the statistics governing the land mine detection problem using EMI sensor data, the GLRT and the LRT provide the same performance. In this problem, the GLRT (shown in Section III that it is essentially a matched filter, $\left.z=\sum_{i=1}^{N} r_{i}\left(e^{-\hat{\mu}_{\alpha_{1}} t_{i}}-e^{\hat{\mu}_{\alpha_{0}} t_{i}}\right)\right)$ always provides the same performance as that of the LRT as long as the statistics of the decay rates of targets and clutter follow certain assumptions.

1) Proposition: Assume the multichannel EMI sensor response is modeled as $\mathrm{r}=e^{-\alpha_{n} \mathbf{t}}$, where $n=1$ or 0 , representing $H_{1}$ and $H_{0}$, respectively, $\mathbf{t}$ is an $N$ by one vector of the sampling times, $\alpha_{1}$ and $\alpha_{0}$ are truncated Gaussian-distributed random variables with means $\mu_{\alpha_{1}}$ and $\mu_{\alpha_{0}}$, and variances $\sigma_{\alpha_{1}}^{2}$ and $\sigma_{\alpha_{0}}^{2}$, and left truncated points of $\alpha_{1}^{l}, \alpha_{0}^{l}$, and right truncated points of $\alpha_{1}^{u}, \alpha_{0}^{u}$, respectively. Furthermore, it is assumed that $\alpha_{0}^{l} \geq \alpha_{1}^{l} \geq 0$ and $\alpha_{0}^{l}<\alpha_{1}^{u} \leq \alpha_{0}^{u}$, based on the underlying physics illustrated in Section II. The limits of $\alpha_{1}^{u}, \alpha_{0}^{u}$ are infinity. The means and variances of $\alpha_{1}$ and $\alpha_{0}$ satisfy $\mu_{\alpha_{1}}<\mu_{\alpha_{0}}$, $\left(\mu_{\alpha_{0}} / \mu_{\alpha_{1}}\right) \geq\left(\sigma_{\alpha_{0}}^{2} / \sigma_{\alpha_{1}}^{2}\right) \geq 1$. Also, assume the sensor is subject to IID Gaussian noise at each sample time. The GLRT on $\mathbf{r}$ is in the form of $z=\sum_{i=1}^{N} r_{i}\left(e^{-\hat{\mu}_{\alpha_{1}} t_{i}}-e^{-\hat{\mu}_{\alpha_{0}} t_{i}}\right)$, and the LRT is of the form shown in (15). Then, the GLRT and LRT are equivalent, thus providing the same performance.

Proof: If it can be shown that $d \lambda / d z \geq 0$, which means the $\operatorname{LR} \lambda$, is a nondecreasing function of the output of a matched filter $z$, and thus, a monotonic function of $z$, the LRT, and the GLRT are equivalent. Because $\lambda$ is not an explicit function of $z$, the chain rule is utilized to prove this relationship

$$
\frac{d \lambda}{d z}=\sum_{i=1}^{N} \frac{\partial \lambda}{\partial r_{i}} \frac{1}{\partial z / \partial r_{i}}
$$

where $r_{i}$ is the received signal from the sensor at the $i$ th sample time at one location, and $N$ is the number of times at which the sensor samples the response. Under $H_{1}$, the output of multichannel time-domain EMI sensor is $r_{i}=e^{-\alpha_{1} t_{i}}+n_{i}(i=$ $1,2, \cdots, N)$, and under $H_{0}$, the sensor output is $r_{i}=e^{-\alpha_{0} t_{i}}+$ $n_{i}$, where $r_{i}$ represents the output from the sensor at the sample time $t_{i}, n_{i}$ is IID white Gaussian noise with zero mean and variance of $\sigma^{2}$. Since the noise terms are IID, the covariance matrix for $\boldsymbol{n}$ is the identity matrix scaled by $\sigma^{2}$.

The LR is then (29), shown at the bottom of the next page, where

$$
\begin{aligned}
& F\left(\mathbf{r}, \alpha_{1}\right)= K_{1} \exp \left[-\sum_{i=1}^{N}\left(r_{i}-e^{-\alpha_{1} t_{i}}\right)^{2} / 2 \sigma^{2}\right] \\
& \cdot \exp \left[-\left(\alpha_{1}-\mu_{\alpha_{1}}\right)^{2} / 2 \sigma_{\alpha_{1}}^{2}\right]
\end{aligned}
$$

and

$$
\begin{aligned}
G\left(\mathrm{r}, \alpha_{0}\right)= & K_{0} \exp \left[-\sum_{i=1}^{N}\left(r_{i}-e^{-\alpha_{0} t_{i}}\right)^{2} / 2 \sigma^{2}\right] \\
& \cdot \exp \left[-\left(\alpha_{0}-\mu_{\alpha_{0}}\right)^{2} / 2 \sigma_{\alpha_{0}}^{2}\right]
\end{aligned}
$$

and

$$
\begin{aligned}
K_{1}^{-1}= & \left(\Phi\left(\frac{\alpha_{1}^{u}-\mu_{\alpha_{1}}}{\sigma_{\alpha_{1}}}\right)-\Phi\left(\frac{\alpha_{1}^{l}-\mu_{\alpha_{1}}}{\sigma_{\alpha_{1}}}\right)\right) \\
& \cdot(2 \pi)^{(N+1) / 2} \sigma^{N} \sigma_{\alpha_{1}} \\
K_{0}^{-1}= & \left(\Phi\left(\frac{\alpha_{0}^{u}-\mu_{\alpha_{0}}}{\sigma_{\alpha_{0}}}\right)-\Phi\left(\frac{\alpha_{0}^{l}-\mu_{\alpha_{0}}}{\sigma_{\alpha_{0}}}\right)\right) \\
& \cdot(2 \pi)^{(N+1) / 2} \sigma^{N} \sigma_{\alpha_{0}}
\end{aligned}
$$

thus, $K_{0}>0$ and $K_{1}>0$. Taking the partial derivative of $\lambda$ with respect to $r_{i}$ yields (32), shown at the bottom of

$$
d^{\prime 2}=\frac{\left(\sum_{i}\left(e^{-\hat{\mu}_{\alpha_{1}} t_{i}}-e^{-\hat{\mu}_{\alpha_{0}} t_{i}}\right)\right)^{2}}{\frac{1}{2}\left[\left(\sum_{i} t_{i} e^{-\hat{\mu}_{\alpha_{1}} t_{i}}\right)^{2} \sigma_{\alpha_{1}}^{2}+\left(\sum_{i} t_{i} e^{-\hat{\mu}_{\alpha_{0}} t_{i}}\right)^{2} \sigma_{\alpha_{0}}^{2}\right]+N \sigma^{2}} .
$$


the page, where the $(\cdots)^{\prime}$ notation denotes a derivative with respect to $r_{i}$. We now write $\partial \lambda / \partial r_{i}=h\left(r_{i}\right) / w(\mathbf{r})$. Since the denominator $w(\mathbf{r})$ is always positive, if $h\left(r_{i}\right)$ is nonnegative, $\partial \lambda / \partial r_{i}$ is nonnegative as well. Based on a theorem presented in [15], the derivative of the integral is equal to the integral of the derivative since the integrand is differentiable with respect to $r_{i}$, i.e.

$$
\left(\int_{\alpha_{1}^{l}}^{\alpha_{1}^{u}} F\left(\mathbf{r}, \alpha_{1}\right) d \alpha_{1}\right)_{r_{i}}^{\prime}=\int_{\alpha_{1}^{l}}^{\alpha_{1}^{u}}\left(F\left(\mathrm{r}, \alpha_{1}\right)\right)_{r_{i}}^{\prime} d \alpha_{1}
$$

Therefore, the numerator is

$$
\begin{aligned}
h\left(r_{i}\right)= & \int_{\alpha_{1}^{l}}^{\alpha_{1}^{u}} \frac{\partial F\left(\mathbf{r}, \alpha_{1}\right)}{\partial r_{i}} d \alpha_{1} \int_{\alpha_{0}^{l}}^{\alpha_{0}^{u}} G\left(\mathbf{r}, \alpha_{0}\right) d \alpha_{0} \\
& -\int_{\alpha_{0}^{l}}^{\alpha_{0}^{u}} \frac{\partial G\left(\mathbf{r}, \alpha_{0}\right)}{\partial r_{i}} d \alpha_{0} \int_{\alpha_{1}^{l}}^{\alpha_{1}^{u}} F\left(\mathbf{r}, \alpha_{1}\right) d \alpha_{1} \\
= & \int_{\alpha_{0}^{l}}^{\alpha_{0}^{u}} \int_{\alpha_{1}^{l}}^{\alpha_{1}^{u}}\left(\frac{\partial F\left(\mathbf{r}, \alpha_{1}\right)}{\partial r_{i}} G\left(\mathbf{r}, \alpha_{0}\right)\right. \\
& \left.-\frac{\partial G\left(\mathbf{r}, \alpha_{0}\right)}{\partial r_{i}} F\left(\mathbf{r}, \alpha_{1}\right)\right) d \alpha_{1} d \alpha_{0} .
\end{aligned}
$$

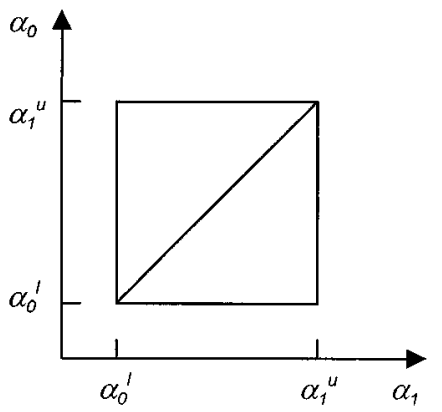

Fig. 2. Integration area for (38). $\alpha_{1}$ and $\alpha_{0}$ are integration variables. The square is divided into upper and lower triangular regions.

TABLE I

NOISE VARIANCE $\sigma^{2}$ REQUIRED TO ACHIEVE $P_{d}=0.7$ AND $P_{f a}=0.1$ FOR FIXED AND RANDOM DECAY RATES

\begin{tabular}{c|c|c}
\hline & Fixed Parameter & Random Parameter \\
\hline Matched Filter & 17.5 & 8.6 \\
\hline Energy Detector & 16.3 & 7.3 \\
\hline Integral detector & 10.8 & 4.9 \\
\hline Threshold Test on & 7.9 & 3.7 \\
Single Time Sample & &
\end{tabular}

$$
\begin{aligned}
& \lambda=\frac{\int_{-\infty}^{\infty} p\left(\mathrm{r} \mid H_{1}, \alpha_{1}\right) p\left(\alpha_{1} \mid H_{1}\right) d \alpha_{1}}{\int_{-\infty}^{\infty} p\left(\mathrm{r} \mid H_{0}, \alpha_{0}\right) p\left(\alpha_{0} \mid H_{0}\right) d \alpha_{0}} \\
& \int_{\alpha_{1}^{l}}^{\alpha_{1}^{u}}\left(2 \pi \sigma^{2}\right)^{-N / 2} \exp \left[-\frac{1}{2 \sigma^{2}} \sum_{i=1}^{N}\left[\left(r_{i}-e^{-\alpha_{1} t_{i}}\right)^{2}\right]\right]\left(\Phi\left(\frac{\alpha_{1}^{u}-\mu_{\alpha_{1}}}{\sigma_{\alpha_{1}}}\right)-\Phi\left(\frac{\alpha_{1}^{l}-\mu_{\alpha_{1}}}{\sigma_{\alpha_{1}}}\right)\right)^{-1} \\
& =\frac{\cdot\left(2 \pi \sigma_{\alpha_{1}}^{2}\right)^{-1 / 2} \exp \left[-\frac{1}{2 \sigma_{\alpha_{1}}^{2}}\left(\alpha_{1}-\mu_{\alpha_{1}}\right)^{2}\right] d \alpha_{1}}{\int_{\alpha_{0}^{l}}^{\alpha_{0}^{u}}\left(2 \pi \sigma^{2}\right)^{-N / 2} \exp \left[-\frac{1}{2 \sigma^{2}} \sum_{i=1}^{N}\left[\left(r_{i}-e^{-\alpha_{0} t_{i}}\right)^{2}\right]\right]\left(\Phi\left(\frac{\alpha_{0}^{u}-\mu_{\alpha_{0}}}{\sigma_{\alpha_{0}}}\right)-\Phi\left(\frac{\alpha_{0}^{l}-\mu_{\alpha_{0}}}{\sigma_{\alpha_{0}}}\right)\right)^{-1}} \\
& \cdot\left(2 \pi \sigma_{\alpha_{0}}^{2}\right)^{-1 / 2} \exp \left[-\frac{1}{2 \sigma_{\alpha_{0}}^{2}}\left(\alpha_{0}-\mu_{\alpha_{0}}\right)^{2}\right] d \alpha_{0} \\
& =\frac{\int_{\alpha_{1}^{l}}^{\alpha_{1}^{u}} F\left(\mathbf{r}, \alpha_{1}\right) d \alpha_{1}}{\int_{\alpha_{0}^{l}}^{\alpha_{0}^{u}} G\left(\mathbf{r}, \alpha_{0}\right) d \alpha_{0}}
\end{aligned}
$$

$$
\frac{\partial \lambda}{\partial r_{i}}=\frac{\left(\int_{\alpha_{1}^{l}}^{\alpha_{1}^{u}} F\left(\mathbf{r}, \alpha_{1}\right) d \alpha_{1}\right)_{r_{i}}^{\prime} \int_{\alpha_{0}^{l}}^{\alpha_{0}^{u}} G\left(\mathbf{r}, \alpha_{0}\right) d \alpha_{0}-\int_{\alpha_{1}^{l}}^{\alpha_{1}^{u}} F\left(\mathbf{r}, \alpha_{1}\right) d \alpha_{1}\left(\int_{\alpha_{0}^{l}}^{\alpha_{0}^{u}} G\left(\mathbf{r}, \alpha_{0}\right) d \alpha_{0}\right)_{r_{1}}^{\prime}}{\left[\int_{\alpha_{0}^{l}}^{\alpha_{0}^{u}} G\left(\mathbf{r}, \alpha_{0}\right) d \alpha_{0}\right]^{2}}
$$




\section{Simulation of fixed decay rate for target and clutter}

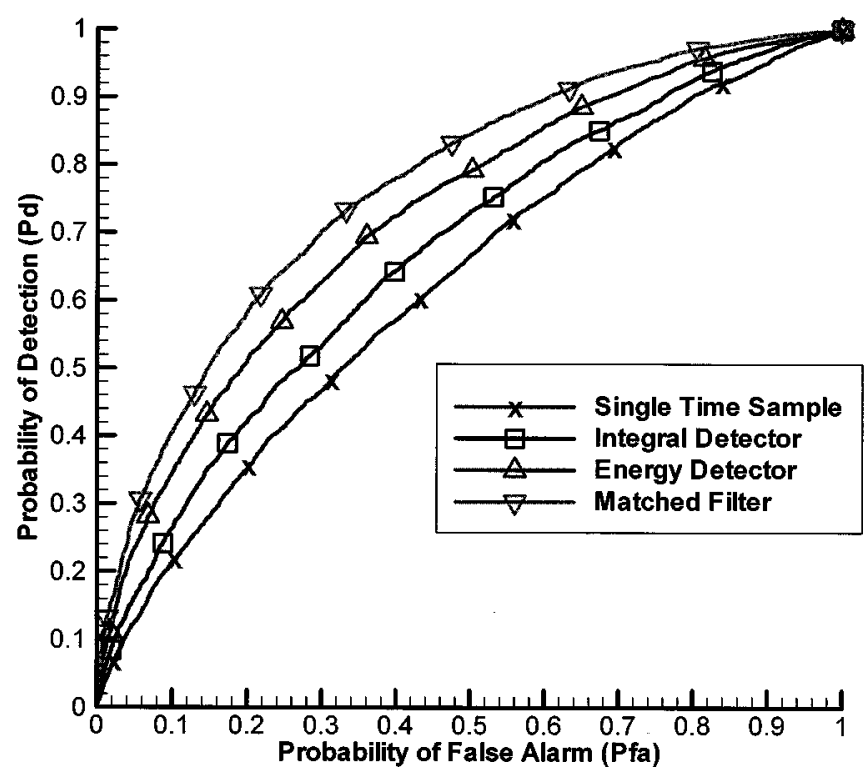

Fig. 3. ROC of simulations of various detection algorithms for a multichanne time-domain EMI sensor at a fixed decay rate for both target and clutter. The decay rate of the target is $\alpha_{1}=2.1$, the decay rate for clutter is $\alpha_{0}=2.5$, and the initial magnitude is 220 for both target and clutter. Gradient: matched filter; delta: energy detector; square: integral detector; cross: threshold test on single time sample.

Taking the derivative of $F\left(\mathbf{r}, \alpha_{1}\right)$ with respect to $r_{i}$

$$
\begin{aligned}
\frac{\partial F\left(\mathbf{r}, \alpha_{1}\right)}{\partial r_{i}}= & K_{1} \sigma^{2}\left(e^{-\alpha_{1} t_{i}}-r_{i}\right) \\
& \cdot \exp \left[-\frac{1}{2 \sigma^{2}}\left[\sum_{j=1}^{N}\left(r_{j}-e^{-\alpha_{1} t_{j}}\right)^{2}\right]\right] \\
& \cdot \exp \left[-\frac{\left(\alpha_{1}-\mu_{\alpha_{1}}\right)^{2}}{2 \sigma_{\alpha_{1}}^{2}}\right] .
\end{aligned}
$$

Similarly

$$
\begin{aligned}
\frac{\partial G\left(\mathbf{r}, \alpha_{0}\right)}{\partial r_{i}}= & K_{0} \sigma^{2}\left(e^{-\alpha_{0} t_{i}}-r_{i}\right) \\
& \cdot \exp \left[-\frac{1}{2 \sigma^{2}}\left[\sum_{j=1}^{N}\left(r_{j}-e^{-\alpha_{0} t_{j}}\right)^{2}\right]\right] \\
& \cdot \exp \left[-\frac{\left(\alpha_{0}-\mu_{\alpha_{0}}\right)^{2}}{2 \sigma_{\alpha_{0}}^{2}}\right] .
\end{aligned}
$$

After combining constants, $h\left(r_{i}\right)$ can be expressed as

$$
\begin{aligned}
h\left(r_{i}\right)= & K_{0} K_{1} \sigma^{2} \int_{\alpha_{0}^{l}}^{\alpha_{0}^{u}} \int_{\alpha_{1}^{l}}^{\alpha_{1}^{u}} \\
& \cdot \exp \left[-\frac{1}{2 \sigma^{2}}\left[\sum_{j=1}^{N}\left(r_{j}-e^{-\alpha_{1} t_{j}}\right)^{2}\right]\right] \\
& \cdot \exp \left[-\frac{1}{2 \sigma^{2}}\left(r_{j}-e^{-\alpha_{0} t_{j}}\right)^{2}\right] \\
& \cdot \exp \left[-\frac{\left(\alpha_{1}-\mu_{\alpha_{1}}\right)^{2}}{2 \sigma_{\alpha_{1}}^{2}}\right] \exp \left[-\frac{\left(\alpha_{0}-\mu_{\alpha_{0}}\right)^{2}}{2 \sigma_{\alpha_{0}}^{2}}\right] \\
& \times\left(e^{-\alpha_{1} t_{i}}-e^{-\alpha_{0} t_{i}}\right) d \alpha_{1} d \alpha_{0} .
\end{aligned}
$$

\section{Simulation of uncertain decay rate for target and clutter}

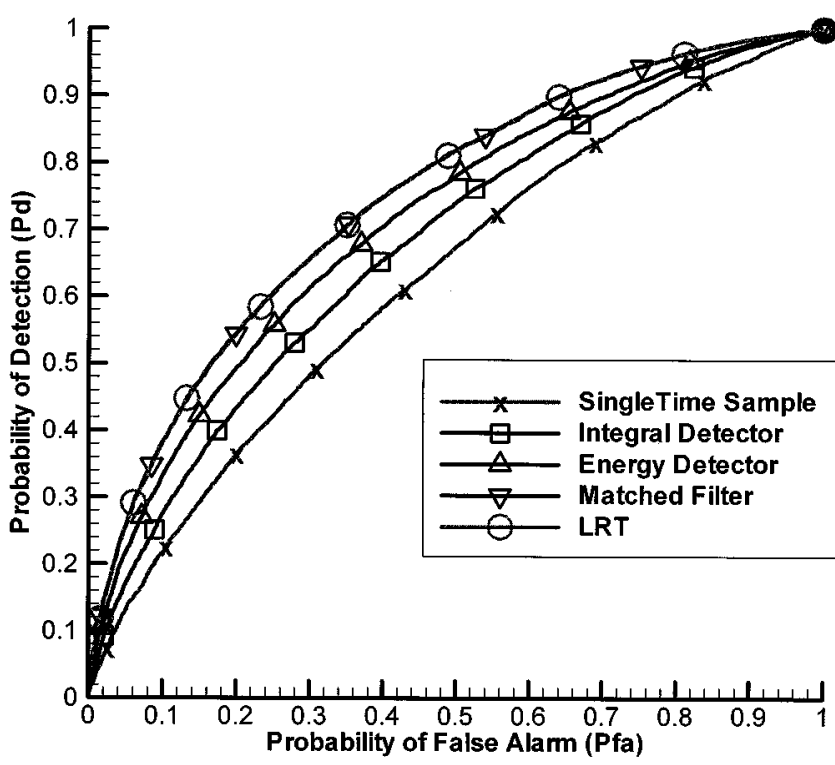

Fig. 4. ROC of simulations of various detection algorithms for multichannel time-domain EMI sensor data with uncertain decay rate for both target and clutter. Distribution of decay rate $\alpha_{1}$ is $N\left(2.1,0.2^{2}\right)$ and for $\alpha_{0}$ is $N\left(2.5,0.2^{2}\right)$. The initial magnitude is 220 for both target and clutter. Circle: the LRT; gradient: matched filter, delta: energy detector; square: integral detector; cross: threshold test on single time sample.

\section{Simulation of uncertain initial magnitude $\&$ decay rate}

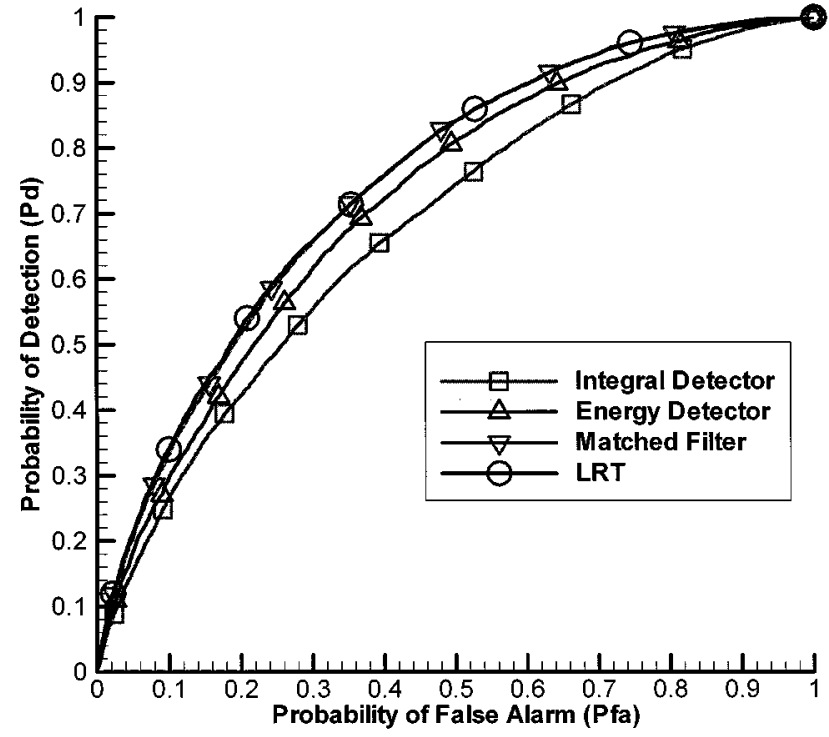

Fig. 5. ROC of simulations of various detection algorithms for multichannel time-domain EMI sensor data with uncertain decay rate and uncertain initial magnitude of target and clutter. Distribution of decay rate $\alpha_{1}$ is $N\left(2.1,0.2^{2}\right)$ and for $\alpha_{0}$ is $N\left(2.5,0.2^{2}\right)$. The distribution of the initial magnitude for target $A$, is $N\left(220,20^{2}\right)$ and for clutter $B$ is distributed as $N\left(210,40^{2}\right)$. Circle: LRT; gradient line: matched filter, delta: energy detector; square: integral detector.

Because exponential functions are always positive, the first four terms of the integrand of the integral in (37) are always positive. The constant term associated with the integral is also positive. Obviously, if the decay rate of the target $\alpha_{1}$ is always slower than that of clutter $\alpha_{0}$, the fifth term in (37), $\left(e^{-\alpha_{1} t_{i}}-\right.$ $\left.e^{-\alpha_{0} t_{i}}\right)$, is also positive, so $h\left(r_{i}\right) \geq 0$. Although in practice, $\alpha_{1}$ 


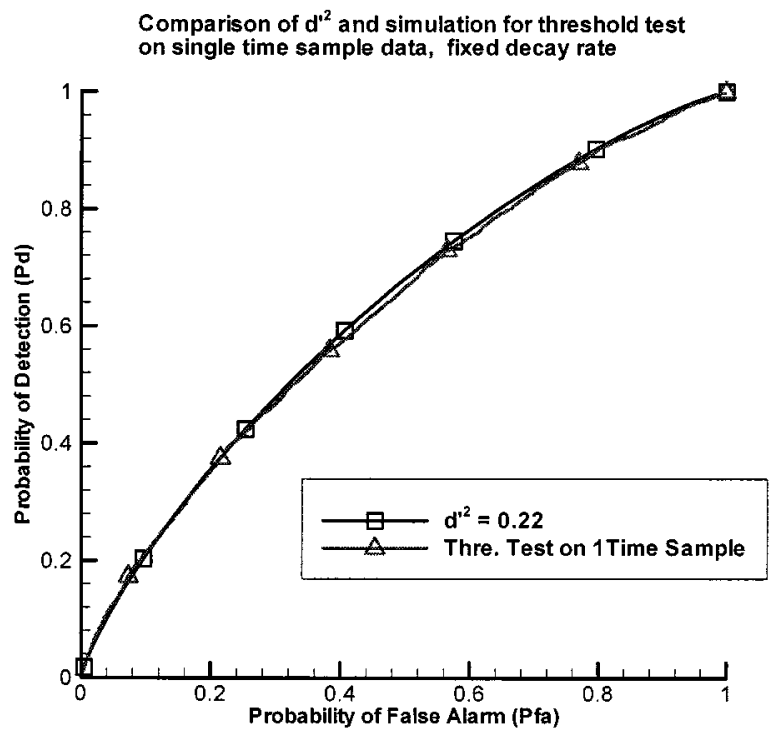

Comparison of $\mathrm{d}^{\prime 2}$ and simulation for energy detector, fixed decay rate

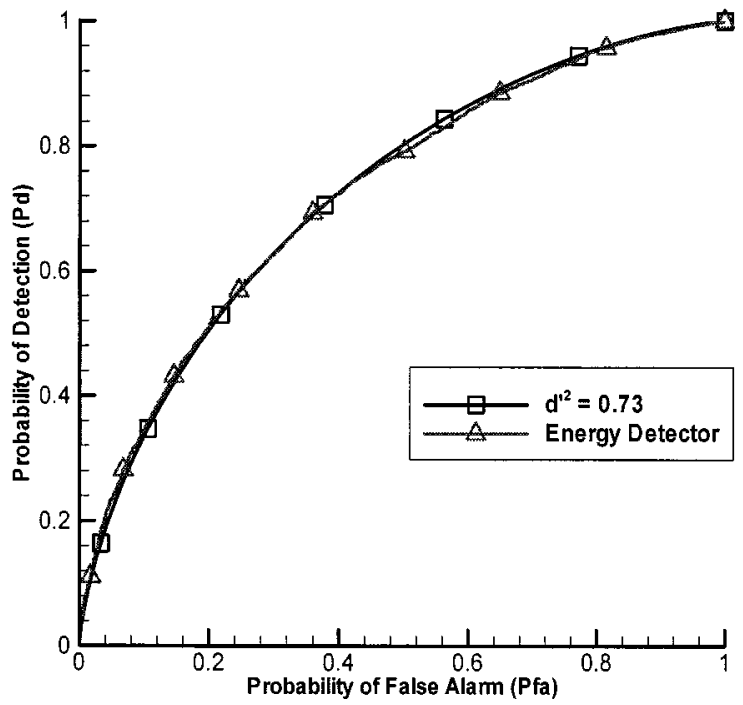

Comparison of $\mathrm{d}^{2}$ and simulation for integral detector, fixed decay rate

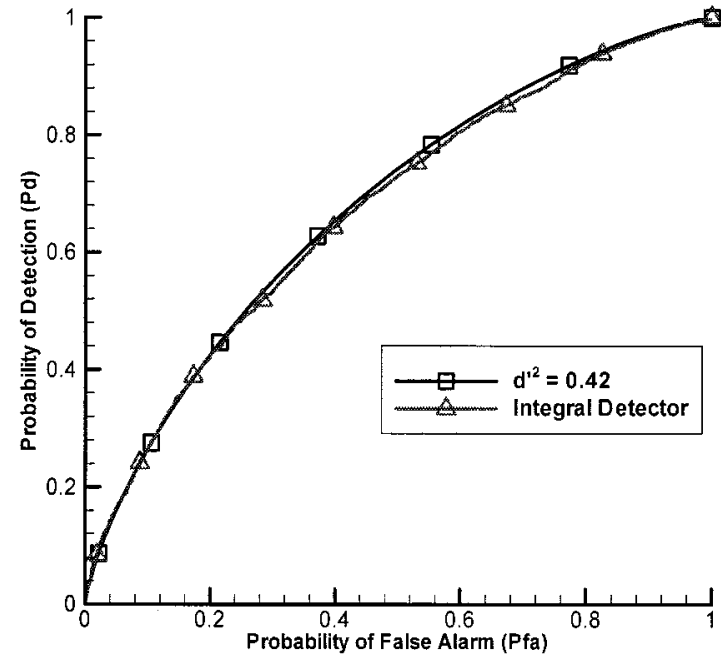

Comparison of $d^{\prime 2}$ and simulation for matched filter, fixed decay rate

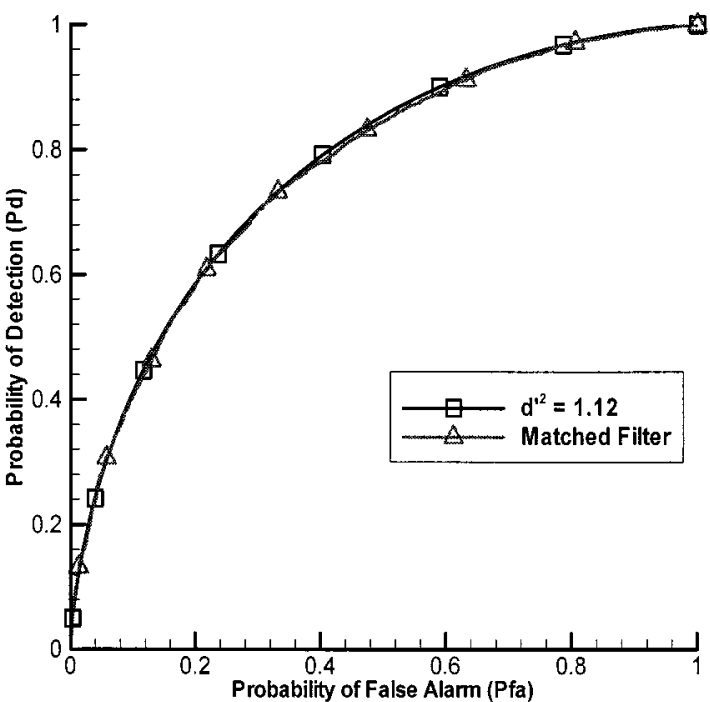

Fig. 6. Comparison of ROC's of the theoretical $d^{\prime}$ value and simulations of various detectors for the fixed decay rate case. (Top left) Threshold test for single time sample time-domain EMI sensor data. (Top right) Integral detector. (Bottom left) Energy detector. (Bottom right) Matched filter for TD EMI sensor data. Delta: simulation; square: theoretical $d^{\prime}$.

TABLE II

THE THEORETICAL VAlues OF $d^{\prime 2}$ FOR VARIOUS PROCESSORS AND KNOWN/RANDOM PARAMETER ENVIRONMENTS

\begin{tabular}{c|c|c}
\hline & Fixed Parameter & Random Parameter \\
\hline Matched Filter & 1.12 & 0.87 \\
\hline Energy Detector & 0.73 & $\approx 0.72$ \\
\hline Integral Detector & 0.42 & 0.38 \\
\hline $\begin{array}{c}\text { Threshold Test on } \\
\text { Single Time Sample }\end{array}$ & 0.22 & $\approx 0.22$ \\
\hline
\end{tabular}

is not always smaller than $\alpha_{0}$, we have assumed the relationships of $\mu_{\alpha_{1}}<\mu_{\alpha_{0}}, \alpha_{1}^{l} \leq \alpha_{0}^{l}<\alpha_{1}^{u} \leq \alpha_{0}^{u}$. The integration area can be divided into four sub-areas, and each sub-area can be integrated separately. Let $K=K_{0} K_{1} \sigma^{2}$. Thus, $K>0$

$$
\begin{aligned}
h\left(r_{i}\right)= & K \int_{\alpha_{0}^{l}}^{\alpha_{1}^{u}}\left\{\int_{\alpha_{1}^{l}}^{\alpha_{0}^{l}}(\cdot) d \alpha_{1}+\int_{\alpha_{0}^{l}}^{\alpha_{1}^{u}}(\cdot) d \alpha_{1}\right\} d \alpha_{0} \\
& +K \int_{\alpha_{1}^{u}}^{\alpha_{0}^{u}}\left\{\int_{\alpha_{1}^{l}}^{\alpha_{0}^{l}}(\cdot) d \alpha_{1}+\int_{\alpha_{0}^{l}}^{\alpha_{1}^{u}}(\cdot) d \alpha_{1}\right\} d \alpha_{0} .
\end{aligned}
$$

It is easily shown that for the first, third, and fourth integrals in (38), the integration is over an area in which $\alpha_{1}$ is always less than $\alpha_{0}$. Thus, integration in these three sub-areas contributes positive or nonnegative value to $h\left(r_{i}\right)$. In the second integral in (38), $\alpha_{1}$ and $\alpha_{0}$ are integrated over an identical region. Let $h_{2}\left(r_{i}\right)$ represent the second integral in (38) after neglecting the 

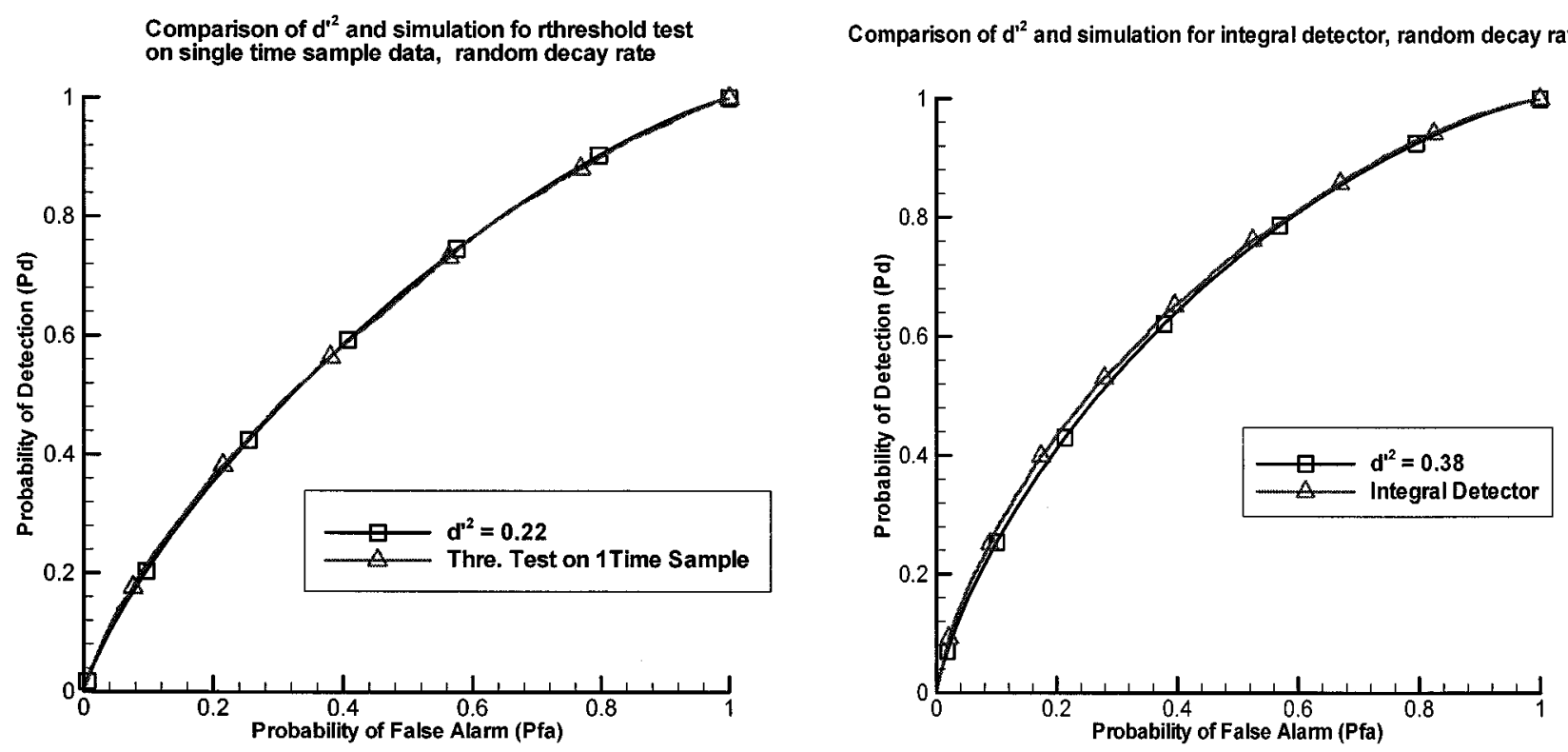

Comparison of $\mathrm{d}^{\mathrm{d}^{2}}$ and simulation for energy detector, random decay rate
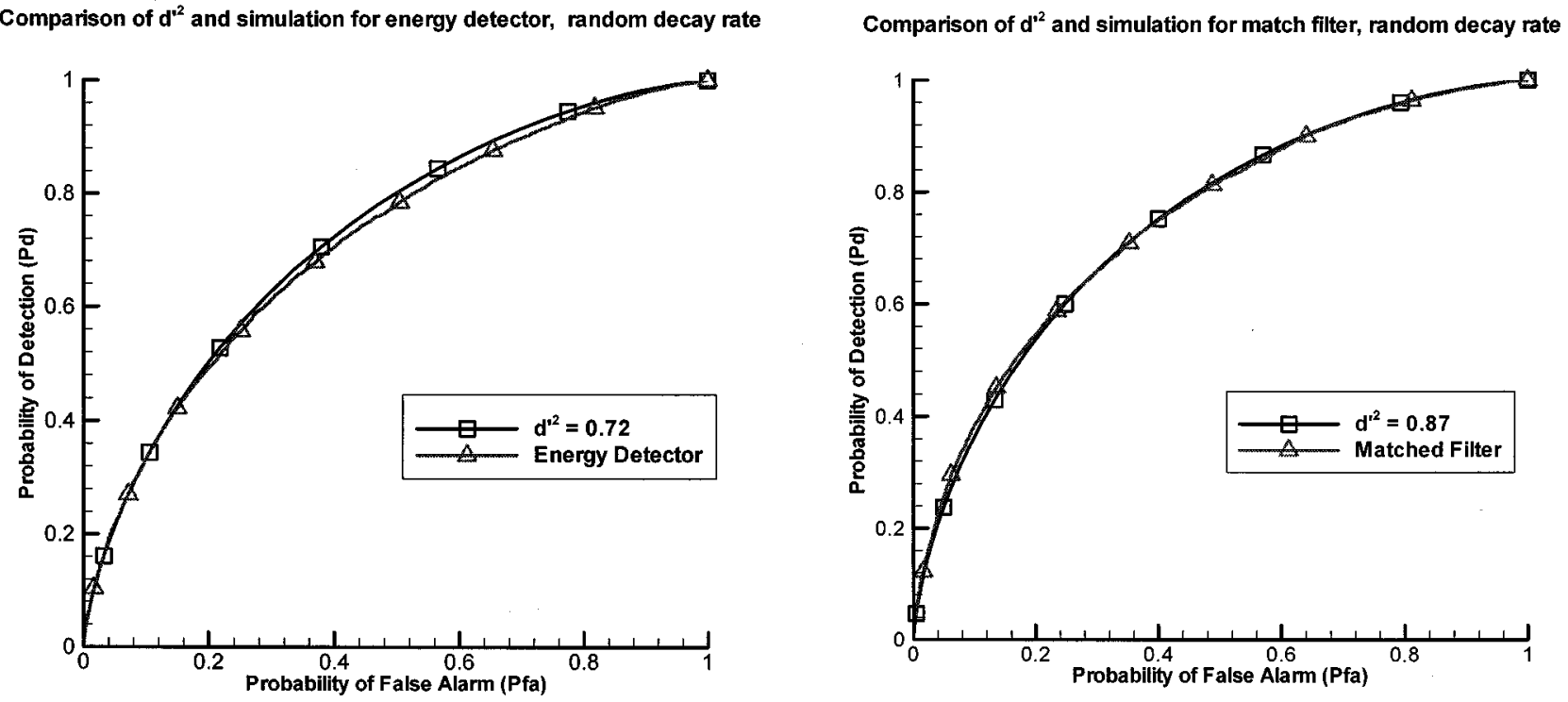

Fig. 7. Comparison of ROC of the theoretical $d^{2}$ value and simulation of various detectors for the random decay rate case. (Top left) Threshold test for single time sample time-domain EMI sensor data. (Top right) Integral detector. (Bottom left) Energy detector. (Bottom right) GLRT/matched filter for time-domain EMI sensor data. Delta: simulation; square: theoretical $d^{\prime}$.

positive constant $K$. The integration area can be further divided into upper triangular and lower triangular portions (Fig. 2 illustrates how the integration areas are divided.) Therefore, $h_{2}\left(r_{i}\right)$ can be written as

$$
\begin{aligned}
h_{2}\left(r_{i}\right) & \int_{\alpha_{0}^{l}}^{\alpha_{1}^{u}} \int_{\alpha_{0}^{l}}^{\alpha_{0}}\left(e^{-\alpha_{1} t_{i}}-e^{-\alpha_{0} t_{i}}\right) \\
\cdot \exp & {\left[-\frac{1}{2 \sigma^{2}}\left[\sum_{j=1}^{N}\left(r_{j}-e^{-\alpha_{1} t_{j}}\right)^{2}\right]\right] } \\
\cdot \exp & {\left[-\frac{1}{2 \sigma^{2}}\left[\sum_{j=1}^{N}\left(r_{j}-e^{-\alpha_{0} t_{j}}\right)^{2}\right]\right] }
\end{aligned}
$$

$$
\begin{aligned}
& \cdot \exp \left[-\frac{\left(\alpha_{1}-\mu_{\alpha_{1}}\right)^{2}}{2 \sigma_{\alpha_{1}}^{2}}\right] \exp \left[-\frac{\left(\alpha_{0}-\mu_{\alpha_{0}}\right)^{2}}{2 \sigma_{\alpha_{0}}^{2}}\right] d \alpha_{1} d \alpha_{0} \\
& +\int_{\alpha_{0}^{l}}^{\alpha_{1}^{u}} \int_{\alpha_{0}^{l}}^{\alpha_{1}}\left(e^{-\alpha_{1} t_{i}}-e^{-\alpha_{0} t_{i}}\right) \\
& \cdot \exp \left[-\frac{1}{2 \sigma^{2}}\left[\sum_{j=1}^{N}\left(r_{j}-e^{-\alpha_{1} t_{j}}\right)^{2}\right]\right] \\
& \cdot \exp \left[-\frac{1}{2 \sigma^{2}}\left[\sum_{j=1}^{N}\left(r_{j}-e^{-\alpha_{0} t_{j}}\right)^{2}\right]\right] \\
& \cdot \exp \left[-\frac{\left(\alpha_{1}-\mu_{\alpha_{1}}\right)^{2}}{2 \sigma_{\alpha_{1}}^{2}}\right] \exp \left[-\frac{\left(\alpha_{0}-\mu_{\alpha_{0}}\right)^{2}}{2 \sigma_{\alpha_{0}}^{2}}\right] d \alpha_{0} d \alpha_{1} .
\end{aligned}
$$


Then, keeping the first integral in (39) the same and exchanging variables $\alpha_{1}$ and $\alpha_{0}$ via a change of variables in the second integral in (39), and changing the integration limits correspondingly, $h_{2}\left(r_{i}\right)$ becomes

$$
\begin{aligned}
& h_{2}\left(r_{i}\right) \\
& =\int_{\alpha_{0}^{l}}^{\alpha_{1}^{u}} \int_{\alpha_{0}^{l}}^{\alpha_{0}}\left(e^{-\alpha_{1} t_{i}}-e^{-\alpha_{0} t_{i}}\right) \\
& \cdot \exp \left[-\frac{1}{2 \sigma^{2}}\left[\sum_{j=1}^{N}\left(r_{j}-e^{-\alpha_{1} t_{j}}\right)^{2}\right]\right] \\
& \cdot \exp \left[-\frac{1}{2 \sigma^{2}}\left[\sum_{j=1}^{N}\left(r_{j}-e^{-\alpha_{0} t_{j}}\right)^{2}\right]\right] \\
& \cdot \exp \left[-\frac{\left(\alpha_{1}-\mu_{\alpha_{1}}\right)^{2}}{2 \sigma_{\alpha_{1}}^{2}}\right] \exp \left[-\frac{\left(\alpha_{0}-\mu_{\alpha_{0}}\right)^{2}}{2 \sigma_{\alpha_{0}}^{2}}\right] d \alpha_{1} d \alpha_{0} \\
& +\int_{\alpha_{0}^{l}}^{\alpha_{1}^{u}} \int_{\alpha_{0}^{l}}^{\alpha_{0}}\left(e^{-\alpha_{0} t_{i}}-e^{-\alpha_{1} t_{i}}\right) \\
& \cdot \exp \left[-\frac{1}{2 \sigma^{2}}\left[\sum_{j=1}^{N}\left(r_{j}-e^{-\alpha_{1} t_{j}}\right)^{2}\right]\right] \\
& \cdot \exp \left[-\frac{1}{2 \sigma^{2}}\left[\sum_{j=1}^{N}\left(r_{j}-e^{-\alpha_{0} t_{j}}\right)^{2}\right]\right] \\
& \cdot \exp \left[-\frac{\left(\alpha_{0}-\mu_{\alpha_{1}}\right)^{2}}{2 \sigma_{\alpha_{1}}^{2}}\right] \exp \left[-\frac{\left(\alpha_{1}-\mu_{\alpha_{0}}\right)^{2}}{2 \sigma_{\alpha_{0}}^{2}}\right] d \alpha_{1} d \alpha_{0} \\
& \int_{\alpha_{0}^{l}}^{\alpha_{1}^{u}} \int_{\alpha_{0}^{l}}^{\alpha_{0}}\left(e^{-\alpha_{1} t_{i}}-e^{-\alpha_{0} t_{i}}\right) \\
& \cdot \exp \left[-\frac{1}{2 \sigma^{2}}\left[\sum_{j=1}^{N}\left(r_{j}-e^{-\alpha_{1} t_{j}}\right)^{2}\right]\right] \\
& \cdot \exp \left[-\frac{1}{2 \sigma^{2}}\left[\sum_{j=1}^{N}\left(r_{j}-e^{-\alpha_{0} t_{j}}\right)^{2}\right]\right] \\
& \cdot\left[\exp \left[-\frac{\left(\alpha_{1}-\mu_{\alpha_{1}}\right)^{2}}{2 \sigma_{\alpha_{1}}^{2}}\right] \exp \left[-\frac{\left(\alpha_{0}-\mu_{\alpha_{0}}\right)^{2}}{2 \sigma_{\alpha_{0}}^{2}}\right]\right. \\
& -\exp \left[-\frac{\left(\alpha_{0}-\mu_{\alpha_{1}}\right)^{2}}{2 \sigma_{\alpha_{1}}^{2}}\right] \\
& \left.\cdot \exp \left[-\frac{\left(\alpha_{1}-\mu_{\alpha_{0}}\right)^{2}}{2 \sigma_{\alpha_{0}}^{2}}\right]\right] d \alpha_{1} d \alpha_{0} \text {. }
\end{aligned}
$$

In (40), the integration is in the upper triangular portion, where $\alpha_{1}<\alpha_{0}$, so $e^{-\alpha_{1} t_{i}}-e^{-\alpha_{0} t_{i}}>0$. The second and third terms of the integrand of (40) are exponential functions. Therefore, they are always positive, and the fourth term, represented by $v\left(\alpha_{1}, \alpha_{0}\right)$, can be simplified to

$$
\begin{aligned}
v\left(\alpha_{1}, \alpha_{0}\right)= & \exp \left[-\frac{\mu_{\alpha_{1}}^{2}}{\sigma_{\alpha_{1}}^{2}}-\frac{\mu_{\alpha_{0}}^{2}}{\sigma_{\alpha_{0}}^{2}}\right] \\
& \cdot\left[\exp \left[-\frac{\alpha_{1}^{2}-2 \mu_{\alpha_{1}} \alpha_{1}}{2 \sigma_{\alpha_{1}}^{2}}-\frac{\alpha^{2}-2 \mu_{\alpha_{0}} \alpha_{0}}{2 \sigma_{\alpha_{0}}^{2}}\right]\right. \\
& \left.-\exp \left[-\frac{\alpha_{0}^{2}-2 \mu_{\alpha_{1}} \alpha_{0}}{2 \sigma_{\alpha_{1}}^{2}}-\frac{\alpha_{1}^{2}-2 \mu_{\alpha_{0}} \alpha_{1}}{2 \sigma_{\alpha_{0}}^{2}}\right]\right]
\end{aligned}
$$

$$
\begin{aligned}
= & \exp \left[-\frac{\mu_{\alpha_{1}}^{2}}{\sigma_{\alpha_{1}}^{2}}-\frac{\mu_{\alpha_{0}}^{2}}{\sigma_{\alpha_{0}}^{2}}\right] \\
& \cdot\left[\exp \left[-\frac{\alpha_{1}^{2} \sigma_{\alpha_{0}}^{2}+\alpha_{0}^{2} \sigma_{\alpha_{1}}^{2}}{2 \sigma_{\alpha_{1}}^{2} \sigma_{\alpha_{0}}^{2}}\right]\right. \\
& \exp \left[\frac{\mu_{\alpha_{0}} \alpha_{0} \sigma_{\alpha_{1}}^{2}+\mu_{\alpha_{1}} \alpha_{1} \sigma_{\alpha_{0}}^{2}}{\sigma_{\alpha_{1}}^{2} \sigma_{\alpha_{0}}^{2}}\right] \\
& -\exp \left[-\frac{\alpha_{1}^{2} \sigma_{\alpha_{1}}^{2}+\alpha_{0}^{2} \sigma_{\alpha_{0}}^{2}}{2 \sigma_{\alpha_{1}}^{2} \sigma_{\alpha_{0}}^{2}}\right] \\
& \left.\cdot \exp \left[\frac{\mu_{\alpha_{1}} \alpha_{0} \sigma_{\alpha_{0}}^{2}+\mu_{\alpha_{0}} \alpha_{1} \sigma_{\alpha_{1}}^{2}}{\sigma_{\alpha_{1}}^{2} \sigma_{\alpha_{0}}^{2}}\right]\right] .
\end{aligned}
$$

If it is assumed $\left(\mu_{\alpha_{0}} / \mu_{\alpha_{1}}\right) \geq\left(\sigma_{\alpha_{0}}^{2} / \sigma_{\alpha_{1}}^{2}\right) \geq 1$, then $\mu_{\alpha_{0}} \sigma_{\alpha_{1}}^{2} \geq$ $\mu_{\alpha_{1}} \sigma_{\alpha_{0}}^{2}$. Multiplying a positive value on each side of the inequality $\left(\alpha_{1} \leq \alpha_{0}\right)$ in the upper triangular integration area for $v\left(\alpha_{1}, \alpha_{0}\right)$, gives $\left(\alpha_{0}-\alpha_{1}\right) \mu_{\alpha_{0}} \sigma_{\alpha_{1}}^{2} \geq\left(\alpha_{0}-\alpha_{1}\right) \mu_{\alpha_{1}} \sigma_{\alpha_{0}}^{2}$. This is equivalent to $\mu_{\alpha_{0}} \alpha_{0} \sigma_{\alpha_{1}}^{2}+\mu_{\alpha_{1}} \alpha_{1} \sigma_{\alpha_{0}}^{2} \geq \mu_{\alpha_{1}} \alpha_{0} \sigma_{\alpha_{0}}^{2}+\mu_{\alpha_{0}} \alpha_{1} \sigma_{\alpha_{1}}^{2}$. Thus

$$
\begin{aligned}
& \exp \left[\frac{\mu_{\alpha_{0}} \alpha_{0} \sigma_{\alpha_{1}}^{2}+\mu_{\alpha_{1}} \alpha_{1} \sigma_{\alpha_{0}}^{2}}{\sigma_{\alpha_{1}}^{2} \sigma_{\alpha_{0}}^{2}}\right] \\
& \quad \geq \exp \left[\frac{\mu_{\alpha_{1}} \alpha_{0} \sigma_{\alpha_{0}}^{2}+\mu_{\alpha_{0}} \alpha_{1} \sigma_{\alpha_{1}}^{2}}{\sigma_{\alpha_{1}}^{2} \sigma_{\alpha_{0}}^{2}}\right]>0 .
\end{aligned}
$$

Also, because $\alpha_{1} \leq \alpha_{0}$ and $\sigma_{\alpha_{1}}^{2} \leq \sigma_{\alpha_{0}}^{2}$, then $\alpha_{1}^{2}\left(\sigma_{\alpha_{0}}^{2}-\sigma_{\alpha_{1}}^{2}\right) \leq$ $\alpha_{0}^{2}\left(\sigma_{\alpha_{0}}^{2}-\sigma_{\alpha_{1}}^{2}\right)$. This yields

$$
\alpha_{1}^{2} \sigma_{\alpha_{0}}^{2}+\alpha_{0}^{2} \sigma_{\alpha_{1}}^{2} \leq \alpha_{0}^{2} \sigma_{\alpha_{0}}^{2}+\alpha_{1}^{2} \sigma_{\alpha_{1}}^{2}
$$

then

$$
\exp \left[-\frac{\alpha_{1}^{2} \sigma_{\alpha_{0}}^{2}+\alpha_{0}^{2} \sigma_{\alpha_{1}}^{2}}{2 \sigma_{\alpha_{0}}^{2} \sigma_{\alpha_{1}}^{2}}\right] \geq \exp \left[-\frac{\alpha_{0}^{2} \sigma_{\alpha_{0}}^{2}+\alpha_{1}^{2} \sigma_{\alpha_{1}}^{2}}{2 \sigma_{\alpha_{0}}^{2} \sigma_{\alpha_{0}}^{2}}\right]>0 .
$$

Therefore, $v\left(\alpha_{1}, \alpha_{0}\right) \geq 0$. Then, $h_{2}\left(r_{i}\right) \geq 0$. To this point, it has been shown rigorously that all the terms in (38) are positive or nonnegative under the assumptions made above. Thus, $h\left(r_{i}\right) \geq$ 0 .

More generally, when $\alpha_{1}^{l}=\alpha_{0}^{l}=0, \alpha_{1}^{u}=\alpha_{0}^{u}=+\infty$, $h\left(r_{i}\right)=h_{2}\left(r_{i}\right) \geq 0$.

At this point, we have proven $\partial \lambda / \partial r_{i} \geq 0$. Next, consider $\partial z / \partial r_{i}$

$$
\frac{\partial z}{\partial r_{i}}=e^{-\hat{\mu}_{\alpha_{1}} t_{i}}-e^{-\hat{\mu}_{\alpha_{0}} t_{i}}>0 .
$$

Given (28), $\partial \lambda / \partial r_{I} \geq 0$ and $\partial z / \partial r_{i} \geq 0$, then $d \lambda / d z$ is nonnegative as well. Therefore, this makes the relationship stated in the proposition proved, i.e., the LRT and the GLRT/matched filter are essentially the same for this problem and provide the same performance.

\section{ReQuirements of NoISE VARIANCE FOR DifFERENT Detectors to ACHIEVE a PARTicular Performance LEVEl}

It is of interest to determine what noise level is required to achieve a particular $P_{d}$ and $P_{f a}$ for different signal processing techniques using the appropriate sensor data. With this motivation, we investigated the required noise variance for each detector in order to achieve a pre-determined performance using multichannel EMI sensor. 
(a) ROC for fixed decay rate case

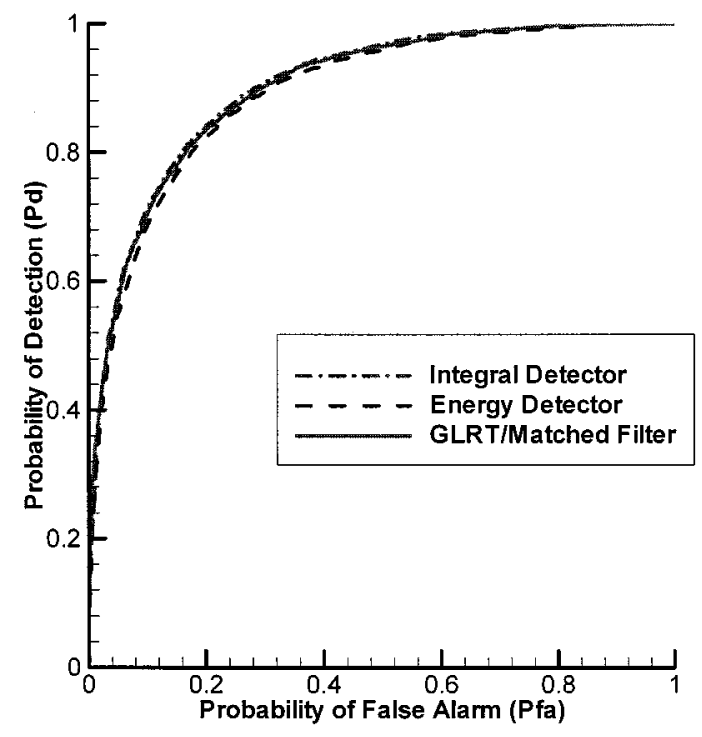

(b) ROC for fixed decay rate case

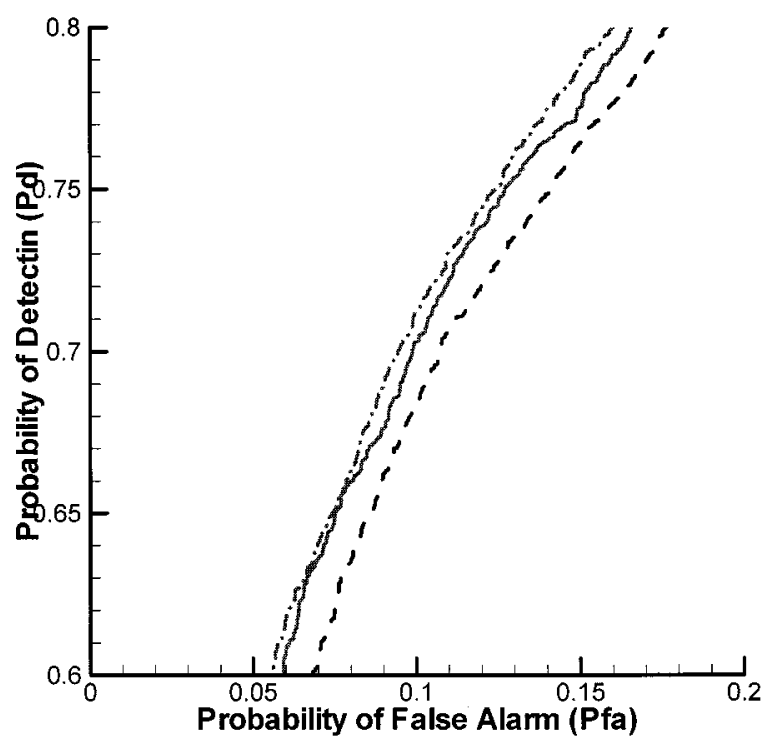

Fig. 8. ROC for various processors at the theoretically calculated noise variance required, achieving $(0.1,0.7)$ performance. (a) Entire scale. (b) Enlarged version of (a).

$P_{d}$ and $P_{f a}$ are defined as [13], [14]

$$
\begin{aligned}
P_{d} & \equiv \int_{\gamma}^{\infty} f\left(x \mid H_{1}\right) d x \\
P_{f a} & \equiv \int_{\gamma}^{\infty} f\left(x \mid H_{0}\right) d x .
\end{aligned}
$$

If the outputs of the detectors follow a Gaussian distribution, it is possible to theoretically calculate the value of noise variance required for known parameters $t, \alpha_{1}$, and $\alpha_{0}$. For the matched filter in the known parameter case, $x$ is Gaussian-distributed as derived above (Section III-A1). The distribution of $x$ under $H_{1}$ is $x \mid H_{1} \sim N\left(\mu_{1}, \sigma_{1}^{2}\right)$, where

$$
\begin{aligned}
& \mu_{1}=\sum_{i}\left(e^{-\alpha_{1} t_{i}}-e^{-\alpha_{0} t_{i}}\right) e^{-\alpha_{1} t_{i}} \\
& \sigma_{1}^{2}=\sum_{i}\left(e^{-\alpha_{1} t_{i}}-e^{-\alpha_{0} t_{i}}\right)^{2} \sigma^{2}
\end{aligned}
$$

and under $H_{0} x \mid H_{0} \sim N\left(\mu_{0}, \sigma_{0}^{2}\right)$, where

$$
\begin{aligned}
& \left.\mu_{0}=\sum_{i}\left(e^{-\alpha_{1} t_{i}}-e^{-\alpha_{0} t_{i}}\right) e^{-\alpha_{0} t_{i}}\right) \\
& \sigma_{0}^{2}=\sum_{i}\left(e^{-\alpha_{1} t_{i}}-e^{-\alpha_{0} t_{i}}\right)^{2} \sigma^{2}
\end{aligned}
$$

so [16]

$$
P_{d}=\int_{\gamma}^{\infty} \frac{1}{\sqrt{2 \pi \sigma_{1}^{2}}} \exp \left[-\frac{\left(x-\mu_{1}\right)^{2}}{2 \sigma_{1}^{2}}\right] d x
$$

Upon letting $y=\left(x-\mu_{1}\right) / \sigma_{1}$

$$
P_{d}=\int_{\left(\gamma \cdot \mu_{1} / \sigma_{1}\right)}^{\infty} \frac{1}{\sqrt{2 \pi}} e^{-\left(y^{2} / 2\right)} d y=\operatorname{cerf}\left(\frac{\gamma-\mu_{1}}{\sigma_{1}}\right)
$$

where $\operatorname{cerf}(\cdot)$ is the complementary error function defined by

$$
\operatorname{cerf}(x)=\int_{x}^{\infty} \frac{1}{\sqrt{2 \pi}} e^{-\left(x^{2} / 2\right)} d x
$$

Similarly

$$
P_{f a}=\operatorname{cerf}\left(\frac{\gamma-\mu_{0}}{\sigma_{0}}\right)
$$

The value of noise variance required to achieve a particular $P_{d}$ and $P_{f a}$ can be obtained by table look-up [16]. For the other detectors, (45) and (46) are still applicable. Corresponding to each detector, appropriate $\mu_{1}, \sigma_{1}^{2}, \mu_{0}$, and $\sigma_{0}^{2}$ values are substituted into (45) and (46).

For the random parameter case, no explicit expression for $P_{d}$ and $P_{f a}$ could be derived, thus no calculation of the theoretical noise variance was made. However, the variance can be estimated through simulation. Table I lists the noise variance required to achieve $P_{f a}=0.1$ and $P_{d}=0.7$ for both a fixed and a random parameter case.

The values in Table I were obtained by using signal $A_{1} e^{-\alpha_{1} \mathbf{t}}$ for $H_{1}$ and $A_{0} e^{\alpha_{0} \mathbf{t}}$ for $H_{0}$. Here, $\mathbf{t}$ is a $20 \times 1$ vector of [.3525, $.4275, .525, .6475, .8025,1.003,1.258,1.583,1.998,2.525$, $3.198,4.055,5.148,6.543,8.323,10.59,13.49,17.19,21.90$, 27.92] measured in $m s$ and $A_{1}=A_{0}=220$ (equivalent to using $\left.A_{1}=A_{0}=1\right)$. In the fixed parameter case, $\alpha_{1}=2.1$, and $\alpha_{0}=2.5$. These values are chosen based on the DARPA experimental data [5]. In the random environment case, $\alpha_{1} \sim$ $\mathcal{N}\left(2.1,0.2^{2}\right)$ and $\alpha_{0} \sim \mathcal{N}\left(2.5,0.2^{2}\right)$. These values and distributions were again chosen based on the statistics of real data. From Table I, it can been concluded that to achieve a particular level of performance for either the fixed or random parameter case, the matched filter can always withstand the highest noise level among the four algorithms investigated. Additionally, an energy detector can achieve the same performance as an integral detector under a higher noise level, or lower SNR. In addition, as we expected if the environment is known exactly, the algorithms can afford lower SNR. In practice, however, the environment is always uncertain. 
(a) ROC for random decay rate case

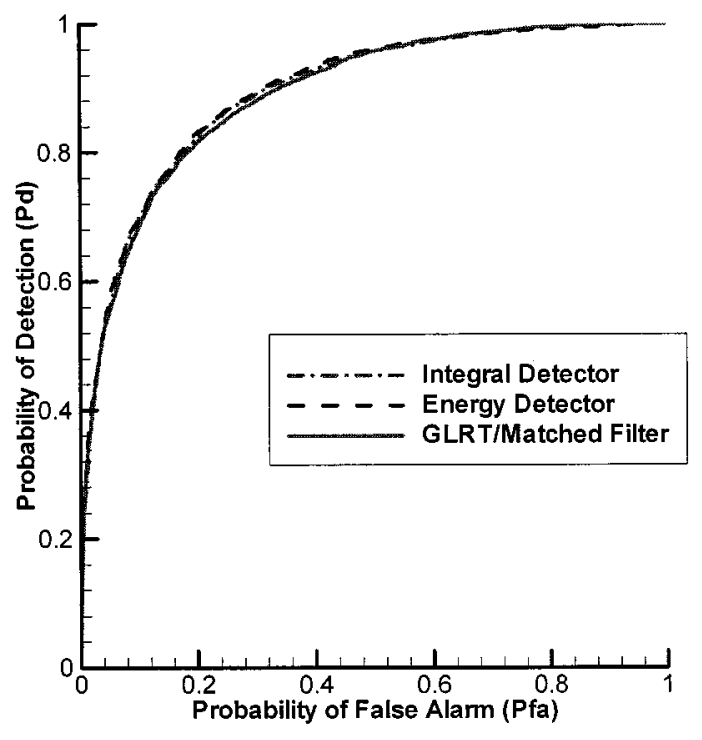

(b) ROC for random decay rate case

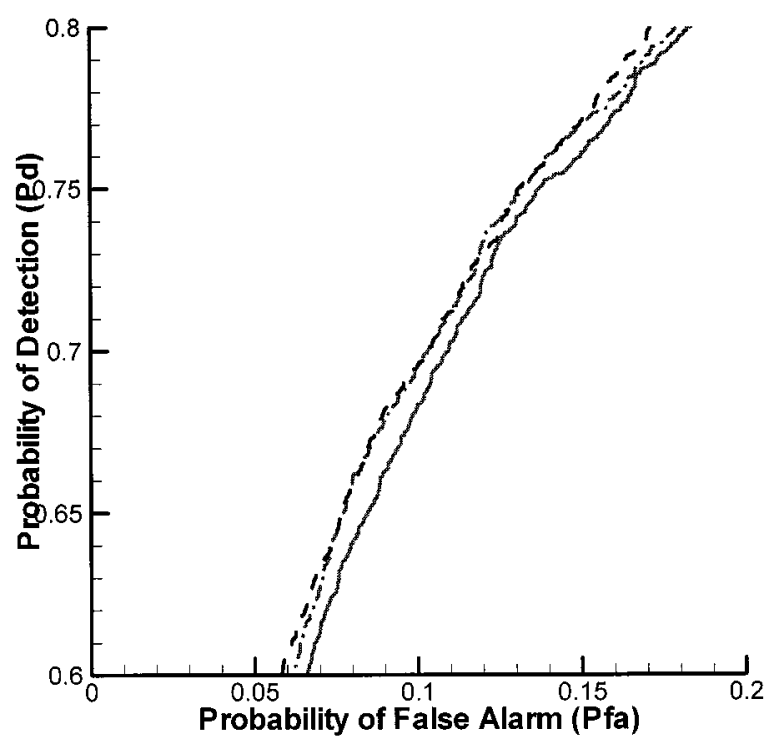

Fig. 9. ROC for various processors at the noise variance estimated from simulations to achieve $(0.1,0.7)$ performance. (a) Entire scale. (b) Enlarged version of (a).

\section{SimULATIONS}

Simulations for EMI sensor data using different detection strategies were implemented. The synthetic data was obtained by adding Gaussian white noise to an exponentially damped signal. In the parameter-known case, the decay rate of the target $\alpha_{1}$ is 2.1 , and that of clutter $\alpha_{0}$ is 2.5 . Those values were chosen by inspecting histograms of the decay rates from targets and clutter obtained from DARPA data, and they are the empirical MLE. For the random parameter case, the distributions of the decay rate and noise variance were also estimated from the DARPA experiment data.

The various signal processing approaches have been implemented on synthetic data. Figs. 3 and 4 show the ROC's for the integral, energy and matched filter detectors and the threshold detector of single time sample data (at the sample time that maximizes the performance) for both fixed parameter and random parameter cases, respectively. As expected, the performance of the LRT/GLRT is better than that of energy detector, which in turn is better than the integral detector, and the performance of threshold detector using single time sample data is the worst among all the detectors considered, since the remaining detectors use multiple time samples of EMI data as their inputs. Additionally, Fig. 4 shows the performance of the LRT when parameters are uncertain, in which Monte Carlo integration was used to compute the integrals in (15). $f\left(r \mid H_{1}\right)=\sum_{k=1}^{M} f\left(r \mid H_{1}, \alpha_{i k}\right) / M$ was used to evaluate the integral in the numerator and denominator of (15), where $i$ is 1 or $0, \alpha_{i k}$ is chosen from Gaussian distributions with means and variances stated above. One thousand iterations were used when calculating the integral.

It is clear that for the random parameter case, the ROC for the LRT is equivalent to that of the matched filter (GLRT), which agrees the theoretical derivation presented in Section III. Furthermore, a simulation obtained when both the decay rate as well as the initial amplitude are random is shown in Fig. 5. Again, the LRT and the GLRT exhibit the same performance.

Next, the theoretical calculation of $d^{2}$ and simulation results for the processors were compared. Table II lists the $d^{\prime 2}$ values for each of the detection algorithms under both fixed and random parameter cases. Figs. 6 and 7 compare the ROC's generated for the Gaussian detection problem with the calculated $d^{\prime 2}$ values and simulation results. ROC's for the theoretical values match the simulation results consistently in all cases. Note that when the variances of the decay rates of target and clutter are small, the uncertainty has almost no effects on the performance.

Again, Table I lists the theoretical noise variance required to achieve $P_{f a}=0.1$ and $P_{d}=0.7$ for the fixed parameter case and the estimated noise variance required to achieve the same performance for the random parameter case. These estimated values were determined by adjusting the noise variance during the simulations. Fig. 8 shows that the ROC curves for the various algorithms at the theoretical required noise variances for the fixed parameter case. It can be seen that the ROC curves approximately cross the point $(0.1,0.7)$, indicating the simulations verify the theoretical calculations. Fig. 9 shows similar ROC curves for random decay rates with the simulated noise.

\section{DISCUSSION}

We have presented results which indicate that a detection theory based approach (LR test) can be used to improve the detectability of land mines and small UXO objects using EMI sensors over standard thresholding techniques. In [3], the results were verified by implementing the processor on real mine and clutter field data, however, only a few targets were present in that case. In this paper, simulation results for each of the processors also verifies the improvement obtained using a LRT detector. Figs. 3 and 4 show that regardless of whether or 
not the parameters are known constants or random variables, an LRT/GLRT/matched filter of multichannel time-domain EMI data always provides the best performance among the algorithms investigated here, and an energy detector performs better than an integral detector. The optimal detector on single time sample of time-domain EMI data is the threshold test, and its performance is worst among all the detectors considered in the paper. This indicates that developing a new sensor which provides more of the time-domain decay curve could prove advantageous for improved landmine detection.

To avoid computational expense, the GLRT is often used as a suboptimal detector. In some cases, the performance of the GLRT can be as good as that of the LRT. In [2], [3], we have shown that for a single-channel (integrated) time-domain EMI sensor, the LRT reduces to the GLRT. In this study, we have shown that for a multichannel time-domain EMI sensor, the GLRT is optimal under certain assumptions on the distributions of the decay rates.

Future work will involve incorporating more environmental parameters into the EMI model. To do so, a more complicated model is required, and a further performance improvement is expected.

\section{ACKNOWLEDGMENT}

The authors would like to thank Dr. L. Carin, Dr. R. Dugan, Dr. T. Altshuler, V. George, Dr. L. Nolte, and Dr. S. Tantum for helpful discussions regarding this research. They are particularly grateful to Dr. A. Barnes for his generous help on the mathematical legality.

\section{REFERENCES}

[1] A. M. Andrews, V. George, and T. W. Altshuler, "Quantifying performance of mine detectors with fewer than 10000 targets," in Proc. SPIE, vol. 3079, Orlando, FL, Apr. 1997, pp. 273-281.

[2] P. Gao, "Improved approaches to land mine remediation using signal detection and estimation theory," M.S. thesis, Duke Univ., Durham, NC, Dec. 1997.

[3] L. M. Collins, P. Gao, and L. Carin, "An improved Bayesian decision theoretic approach for land mine detection," IEEE Trans. Geosci. Remote Sensing, vol. 37, pp. 811-819, Mar. 1999.

[4] P. Gao and L. M. Collins, "Improved signal processing approaches for landmine detection," in Proc. SPIE: Detection Technologies for Mines and Minelike Targets, Orlando, FL, Apr. 1998.

[5] V. George, T. Altshuler, A. Andrew, J. Nicoll, E. Cespedes, D. Butler, T. Broach, and R. Mehta, Background Data Collection Plan, Defense Adv. Res. Projects Agency (DARPA), Def. Sci. Office, Arlington, VA, Dec. 1996.
[6] G. D. Sower and S. P. Cave, "Detection and identification of mines from natural magnetic and electromagnetic resonances," in Proc. SPIE, vol. 2496, Orlando, FL, Apr. 1995, pp. 1015-1024.

[7] N. Geng, P. Garber, L. Collins, L. Carin, D. Hansen, D. Keiswetter, and L. J. Won, "Wideband electromagnetic induction for metal-target identification: Theory, measurement and signal processing," Tech. Rep., Duke Univ., Durham, NC, 1998.

[8] C. E. Baum, "Low-frequency near-field magnetic scattering from highly, but not perfectly, conducting bodies,", Albuquerque, NM, Phillips Lab. Interaction Note 499, Nov. 1993.

[9] Y. Das, J. E. McFee, and R. H. Cherry, "Time-domain response of a sphere in the field of a coil: Theory and experiment," IEEE Trans. Geosci. Remote Sensing, vol. GE-22, pp. 360-367, July 1984.

[10] Y. Das, J. E. McFee, J. Toews, and G. C. Short, "Analysis of an electromagnetic induction detector for real-time location of buried objects," IEEE Trans. Geosci. Remote Sensing, vol. 28, pp. 278-288, May 1990.

[11] A. D. Whalen, Detection of Signal in Noise. New York: Academic, 1971, p. 352.

[12] N. Geng, C. E. Baum, and L. Carin, "On the low-frequency natural response of conducting and permeable targets," IEEE Trans. Geosci. Remote Sensing, vol. 37, pp. 347-359, Jan. 1999.

[13] H. L. Van Trees, Detection, Estimation, and Modulation Theory. New York: Wiley, 1968.

[14] L. Selin, Detection Theory. Princeton, NJ: Princeton Univ. Press, 1965.

[15] G. B. Folland, Real Analysis: Modern Techniques and Their Applications. New York: Wiley, 1984.

[16] M. H. DeGroot, Probability and Statistics. Reading, MA: AddisonWesley, 1989.

Ping Gao (S'97) receved the B.S. and M.E. degrees (honors) in electrical engineering from Beijing University, Beijing, China, in 1993 and 1996, respectively, and the M.S.E.E. from Duke University, Durham, NC, in 1997, where she is currently pursuing the $\mathrm{Ph} . \mathrm{D}$. degree in electrical and computer engineering.

Since 1997, she has been with Duke University as a Research Assistant. Her main research interests are in signal detection and estimation theory, statistical signal modeling and processing, and applied electromagnetics.

Leslie M. Collins (S'83-M'96) was born in Raleigh, NC, in 1963. She received the B.S.E.E. degree from the University of Kentucky, Lexington, in 1985, and the M.S.E.E. and Ph.D. degrees in electrical engineering from the University of Michigan, Ann Arbor, in 1986 and 1995, respectively.

She was a Senior Engineer with Westinghouse Research and Development Center, Pittsburgh, PA, from 1986 to 1990. Since 1995, she has been with the Electrical and Computer Engineering Department, Duke University, Durham, NC. Her research interests include incorporating physics-based models into statistical signal processing algorithms, and she is pursuing applications in subsurface sensing and applied electromagnetics, as well as enhancing speech understanding by hearing impaired individuals.

Dr. Collins is a member of the Tau Beta Pi, Eta Kappa Nu, and Sigma Xi societies. 